C. G. Wohl, R. L. Kelly, F. E. Armstrong C. P. Home,

M.S. Hutchineon, A. Rittenberg, T. G. Trippe, G. P. Yost

Berkeley Particle Data Group

Lawrence Berkeley Laborafory

Berkeley, Califomia 94720, USA

L. Addis

Stanford Linear Accelerator Center

Stanford, California 94305, USA

C. E. W. Ward

Argonne National Laboratory

Argonne, Illinois 60439, USA

N. Beggett

Brookhaven National Laboratory

Uptan, New York 11973, USA

Y. Goldschmidt-Clermont

CERN

CH-1211 Geneva 23, Suitzerland

P. Joos

DESY

Notkestipg 1

D.2 Hamburg-52, Cermany

N. Gelfand

Fermilab

P.O. Bor 500

Bafacia, Illinois 605I0, I'SA

Y. Oyanagi

University of Tsukuba

Sakuro-mura, Nithari-gun

Ibaraki-ken 305, Japan

S. N. Grudtsin, Yu.G. Ryabov Instifute for High Energy Physics Serpukhov, Profvino

Moscow Region, USSR

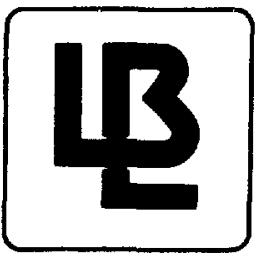

LAWRENCE BERKELEY LABORATORY

University of California

Prepared for the Department of Energy under contract W-7405-ENG-48 


\section{COMPILATION OF CURRENT HIGH-ENERGY PHYSICS EXPERIIENTS}

C.G. Whhl, R.L. Kelly, F.E. Armstrong, C.P. Horne, M.S. Hutchinson, A. Rittenberg, T.G. Trippe, G.P. Yost Berkeley Particle Data Group* Lawrence Berkeley Laboratory

Berkeley, California 94720, USA

L. Addis

St anford Linear Arrelerator Center

St anford, Californiu 94305 , USA

C.E.W. Ward

Argonne National Laboratory

Argonne, Illinois 60439, USA

N. Baggett

Brookhaven National Laboratory

Upton, New York 11973, USA

Y. Goldschmidt-Clermont

CERN

CH-l21l Geneva 23, Switzerland

P. Joos

DESY

Notkestieg 1

D-2 Hamburg-52, Germany

N. GeIfand

Fermilab

P.0. Box 500

Batavia, Illinois 60510 , USA

Y. Oyanagi

University of Tsukuba

Sakura-mura, Niihari-gun

Ibaraki-ken 305, Japan

S. N. Gruitsin, Yu.G. Ryabov

Institute ior High Energy Physics

Serpukhov, Protvino

Moscow Region, USSR

*The Berkeley Particle Data Group is supported by the Director, office of Energy Resea:ch, Office of High Energy and Nuclear Physics, Division of High Energy Physics of the U.S. Department of Energy under Contract No. W-7405-ENG-48 and by the National Science Foundation. 


\section{Introduction}

This is the fourth edition of our compilation of current high energy physics experiments. It is a collaborative effort of the Berkeley Particle Data Group, the SLAC library, and nine participating laboratories: Argonne (ANL), Brookhaven (BNL), CERN, DESY, Fermilab (FNAL), the Institute for Nuclear Study, Tokyo (INS), KEK, Serpukhov (SERP), and SLAC.

The compilation includes summaries of all high energy physirs experiments at the above laboratories that (1) were approved (and not subsequently withdrawn) before about Apr 11 1981, and (2) had not completed taking of data by 1 January 1977. We emphasize that only approved experiments are included.

The contents of the compilation are:

Summaries of experiments -- These are on the microfiche in the pocket at the front of the report. An example from the summaries, with some explanatory notes, follows this introduction.

Indices -- These follow the example. One Index lists experiments by initial-state particles and beam momentum, in order of increasing particle mass and beam momentum. Another index lists experiments by spokesman.

Vocabularies -- These follow the indices, and glve names and abbreviations used in the sumaries. Therp are vocabularies for arcelerators, detectors, particles, institutions, etc.

Beam 1ists -- These list beams for fixed-target experiments at Brookhaven, CERN, Fermilab, KEK, Serpukhov, and SLAC.

Anyone wanting more information about a particular experiment should contact the experiment's spokesman directly, not us. Although the original experimental proposals are sometimes available in librarles, there are often subsequent letters, revisions, and addenda, or simply informal arrangements with the powers that be, that extend the aims or shift the emphasis of an experiment. There are also often changes of collaborators on an experiment. We try to keep up with such changes, but of course cannot entirely succeed. The spokesman is the authoritative source of information about an experiment. 
We invite comments pointing out omissions, obscurities, outof-date information, and outright errors. There are no doubt a number of each. Comments should be sent to:

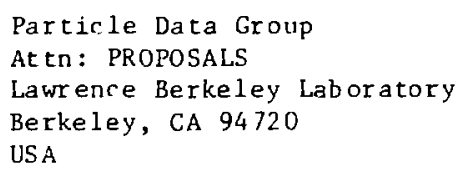

Requests for copies from the Americas, Australasia, and the Far East should go to the above address, while those from other areas should go to:

CERN Scientific Information Service $\mathrm{CH}-1211$ Geneva 23

Swl czerland 


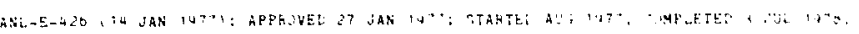

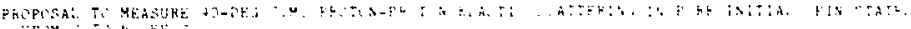

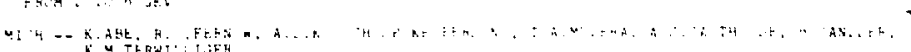

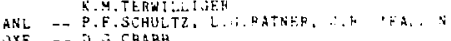

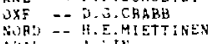

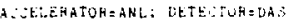

PJIAH:LED HEAM ANI TARGET

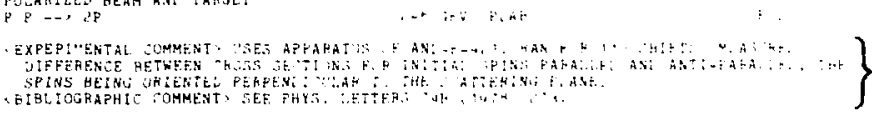

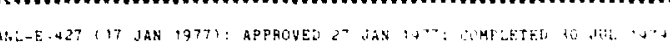

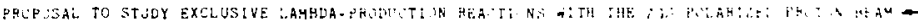

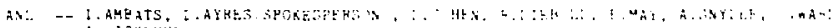

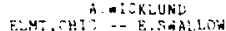

ALLELEFATOR=AK.,; TETE' 'T.JH=EM-

P.LARTZEU TEAY

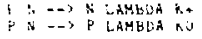

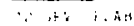

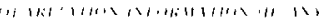

X.NSPECS.

EX, MABS, $2, \dot{X}, \mathrm{X}$

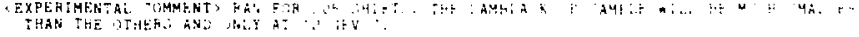

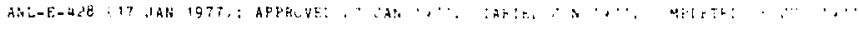

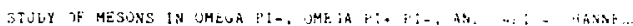

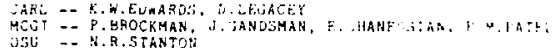

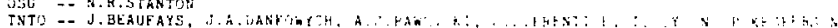

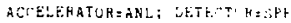

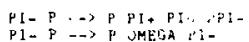

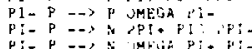

$\because, \because: \quad: A:$

$\begin{array}{ll}\text { di } 1235)- & 235 \\ \text { ij } 11706:-\end{array}$

a 2131010

MESOR (UHSPEC)

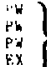

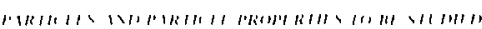

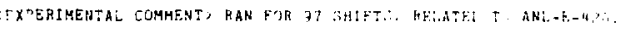

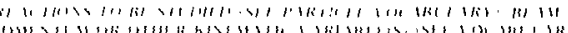

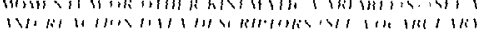


BEAM-TARGET-HONENTUM INDEX

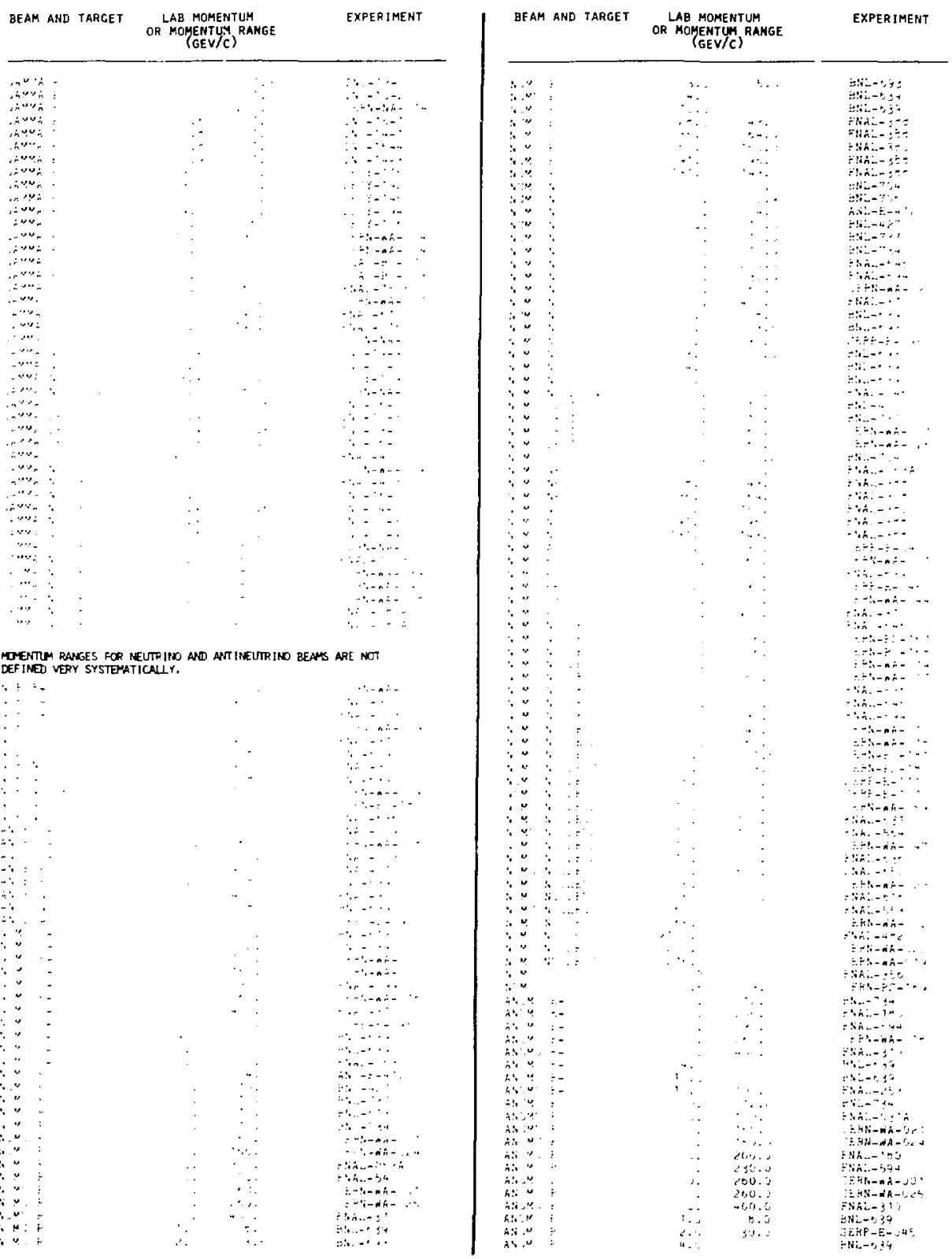


BEAM-TARGET-MONENTUM IHDEX

\begin{tabular}{|c|c|c|c|c|c|c|c|c|}
\hline \multirow{2}{*}{$\frac{\text { BEAH }}{\text { AnLMLi }}$} & \multirow{2}{*}{ AND TAREET } & \multicolumn{2}{|c|}{$\begin{array}{l}\text { LAB MOHENTUM } \\
\text { OF MOHENTL RANGE } \\
\text { (GEV/C) }\end{array}$} & EXPER IMENT & BEAM AND TARGET & $\begin{array}{r}\text { LAB MOMEN } \\
\text { OR MOHENTY } \\
\text { GEV/C }\end{array}$ & UMA & EXPER:MENT \\
\hline & & 10.0 & & BNL -539 & $E+E-$ & $?$ & & SLAC-SP-039 \\
\hline ADUNS & $\mathbf{F}$ & $2 E+0$ & 43.0 & FNAL-388 & $\bar{z}+E-$ & 1,1 & 1.3 & DESY-19 \\
\hline ARUA: & p & 35.0 & 64.0 & FNAL-388 & $E+E-$ & 1.5 & 3.0 & SLAC-SP-028 \\
\hline 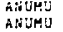 & $\stackrel{p}{p}$ & 50.0 & 150.0 & FNAL- 380 & $E+E-$ & 1.5 & 4.0 & $S L A C-S P-029$ \\
\hline Anter: & $\stackrel{p}{p}$ & $9: 0$ & $\begin{array}{r}95.0 \\
143.0\end{array}$ & FNAL-38a & $E+E-$ & 1.5 & 4.2 & $S L A=S P-024$ \\
\hline ANURU & : & 130 & $\begin{array}{r}143.0 \\
12.0\end{array}$ & $\begin{array}{l}\text { FNAL-3aB } \\
\text { UN:-734 }\end{array}$ & $E+E-$ & 1.5 & 4.2 & $B L A C-5 \%-030$ \\
\hline REUTU & $\mathrm{N}$ & 2. & 200.0 & $\begin{array}{l}\text { ENL-734 } \\
\text { FNA }-180\end{array}$ & $E+E-$ & 1.5 & $4 \cdot 3$ & $\begin{array}{l}\text { DESY }-144 \\
\text { DESY }-119\end{array}$ \\
\hline ANUK: & 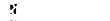 & 0. & 260.0 & $C E P N=W A=025$ & $E+E$ &, 1.0 & & $\begin{array}{l}\text { LESY - } 11 \\
\text { EESY - } 138\end{array}$ \\
\hline ANUTH & $\mathrm{N}$ & 0. & 400.0 & FNAL -310 & $E+E-$ & $1 . \hat{6}$ & 2.5 & $D E S Y-143$ \\
\hline RNUMU & $\mathrm{N}$ & 9.3 & 8.0 & ENL-639 & $E+E-$ & 1.8 & & $2 E S Y-143$ \\
\hline ANUMU & $\mathrm{N}$ & -2.5 & & $B N L-639$ & $E+E-$ & 1.9 & $\bar{c} .5$ & DESY +29 \\
\hline ANUMU & $\mathrm{Ii}$ & 15.5 & & $G N L-639$ & $E+E-$ & $\therefore$ & 2.3 & EESY - THO \\
\hline RRUMU & 2EUT & 0. & $\begin{array}{l}100.0 \\
2 t 0.0\end{array}$ & FNAL -390 & $E+E-$ & 3.7 & $5 . \varepsilon$ & DESY-LENA \\
\hline $\begin{array}{l}\text { ANUMU } \\
\text { ANUMU }\end{array}$ & $\begin{array}{l}\text { QEUT } \\
\text { WEUT }\end{array}$ & 0. & $\begin{array}{l}260.0 \\
260.0\end{array}$ & $\begin{array}{l}C E R N-W A-O C \\
\text { CERN-Wh }=\text { C25 }\end{array}$ & $\begin{array}{l}\bar{E}+E- \\
E+E-\end{array}$ & $\begin{array}{l}2.0 \\
4.0\end{array}$ & $\begin{array}{r}5.0 \\
.8 .0\end{array}$ & $\begin{array}{l}S-746 \\
S L A C-F E P-O C Z\end{array}$ \\
\hline AN UMU & NE & $\mathrm{j}$ & 200.0 & FNAL-:80 & $E+E-$ & -.2 & 16.0 & SLAC-PEP-OOH \\
\hline ANUMU & $\mathrm{NE}$ & 25.0 & 43.0 & FNAL $-38 \mathrm{~A}$ & $E+E-$ & 4.5 & 18.0 & SLAC-PEP-OO5 \\
\hline ANUMU & NE & 35.0 & 64.0 & FNAL-38B & $\ddot{E}+E_{-}$ & 4.5 & $: 8.0$ & SLAC-FEP $=000$ \\
\hline AN SMU & NE & 23.0 & 150.0 & $P N A L-380$ & $E+E-$ & 4.2 & $-\varepsilon, 0$ & $S: A C-P E=00 S$ \\
\hline ANGMU & $\mathrm{HE}$ & $9 \div .0$ & 95.0 & FUAL- JEA & $E+E-$ & 4.2 & $: 0.0$ & $\mathrm{~S}: A=-P E \mathrm{~F}-\mathrm{C} 12$ \\
\hline Allimis & ME & $: 51.0$ & 143.0 & $F: N A L-388$ & $E \cdot \bar{E}-$ & +.2 & $: 3,3$ & SIAE-?ET-214 \\
\hline ANGMU & $A L$ & 5.2 & 30.0 & $S E B P-E-C 45$ & E. E- & +28 & . & $B \perp A C-T E F-j B$ \\
\hline AN:MU & $\vec{z}$ & . & 200.6 & EERH-WA-OS & $E \cdot \bar{E}=$ & 4.2 & 19.3 & $S \angle A=-P E P-O Z O$ \\
\hline ANSWL & $F \bar{E}$ & $\therefore$ & 260.3 & $\therefore E R N-B A-D+8$ & $E+E-$ & 4.5 & 5.4 & CESY-RPGUS \\
\hline ANUMU & $\bar{F} \bar{E}$ & & 1300.6 & $F: A D-69+4$ & $E+E-$ & $\ldots$ & $\leq 1$ & $\therefore \in=Y-: 47$ \\
\hline ANumis & $\mathrm{FE}$ & $c, i$ & 30.0 & $\operatorname{SEh} P-E-945$ & $E_{*}$ & $\therefore 2$ & 26.6 & EESU-PETRA-GEL \\
\hline ANUNU & MUCLE:S & $?$ & & $F N A=546$ & $E+\bar{E}-$ & $\therefore$ & $2 \therefore .0$ & SEJY-PETRA-JASF \\
\hline ANUMU & NUCLEUE & 2 & 6.0 & ERN-FS- & $\bar{E}+\bar{E}-$ & $\overline{\Xi 5}$ & 20.0 & ZESY-PETHA-MAEK \\
\hline ANTHAS & MGCLES & $\therefore$ & 6.5 & ZERH-PS- 169 & $\therefore \cdot E-$ & $\therefore 2$ & $2^{n} \cdot 3$ & CESY-PETRh-PLUTC \\
\hline ANUAL & IUCLEUS & 5. & 200.0 & IRAL- 536 & E. E- & $5 . ?$ & $2 \omega$. & CESY-PETF A-PLUH \\
\hline ARICYI & HUCLEUS & 5. & 230.0 & Fiski. 5034 & $\therefore+$ & $\therefore$ & 26.5 & LEST-PETAR-TASS \\
\hline AR UMl & NUCLESE & 2 & $\angle 50.0$ & Fati-inh-J IE & & & & \\
\hline ANiY' & MUCLE:. & 5.0 & 20.5 & $3 E R P-E-111$ & E. & 5. : & 20.9 & ER+i-ih \\
\hline htISU & HUCLEUE & 13 & 20.3 & $\because A P-E-107$ & M. F & 38 & 256.0 & CEFH-SA-ZCE \\
\hline ลNบนH & NUT EUS & $: 3.8$ & 100.0 & EERA-HA-059 & $4 i-i$ & 30.2 & 25,5 & $=E N-n_{h}-2 r_{S}$ \\
\hline AnTSYIS & NULLEAS & $: 0.5$ & 000.0 & FHA:-5:7 & $M \because-\mu E$ & & & $\sin =7+5$ \\
\hline ANITH' & NUCLEUS & $1 \div 0$ & $100 \%$ & FNA:-554 & $M Z-B E$ & $: 56.6$ & & FrihL-Lan \\
\hline ASTLUU & NJCLES & $\because \ldots$ & 200.6 & $=E P t^{*}-H A-54^{-}$ & $M Z-C !$ & $\therefore 5.5$ & & Fshi-4th \\
\hline Alion: & MUCLEUS & $\angle \lambda$ & $0,50.0$ & $F: B 2 i-052$ & 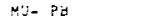 & $\therefore$ & & $F: A i=\cdots j$ \\
\hline intuli & NuCLEUE & $\therefore:$ & $\cos \theta$ & FHAL-5. 16 & MIJ- NUCLEUS & $\because 5$ & $250=$ & ESH-1BA- i, \\
\hline An:M j & MUCLEUS & $\because \because ?$ & & $5.4 A_{-}-5,53$ & $M U+A L$ & $\because:$ & & $8 \div-74-$ \\
\hline A.: $=M$ & NUC:E'ZZ & $, \because \therefore:$ & & $F: k_{i}-4+2$ & $M j+F \ddot{z}$ & i & & $3+n_{-}-25=\dot{1}$ \\
\hline Axosis & MC:Eบ & $\therefore \quad \cdots$ & & EPR-AR-TIY & 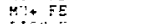 & ist. & & $\because A_{-}=09$ \\
\hline tot? & $y=$ & . $\quad \vdots$ & $\therefore$ … & 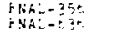 & ELA E- & & & $\therefore A R-N A-O B$ \\
\hline$\therefore-$ & & 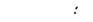 & & $\therefore A B-=-\angle Z$ & $3 i+3 i-$ & C. & & $\therefore-\dot{r}-\bar{E}-\overline{-}$ \\
\hline$=-$ & & $\therefore$ & & $\begin{array}{ll}3 \\
\therefore E S-5-13\end{array}$ & 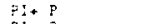 & $?$ & & $\therefore \therefore-6-1+1$ \\
\hline$t-t$ & & $\therefore$ & & 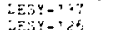 & 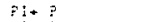 & $\because$ & $\therefore:$ & $\therefore+\because-P+-\cdots$ \\
\hline $\begin{array}{l}=5 \\
=-5\end{array}$ & & $\therefore$ & $\because \vdots$ & 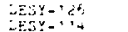 & $3:+5$ & $\because$ & $\therefore$. & $\because+\ldots-\cdots$ \\
\hline 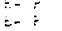 & & $\because \ldots$ & & 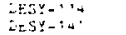 & 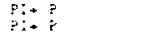 & $\because$ & & $=2:-\div$ \\
\hline$E-$ & & $\therefore$ & & $\begin{array}{l}\text { iesy-ius } \\
\text { Esy-1:- }\end{array}$ & $\begin{array}{l}3: 8 \\
3:+8\end{array}$ & $\because \cdots$ & $<$. & $\therefore \div=-Z^{\circ}<$ \\
\hline$z-\vdots$ & & $\therefore$ & & 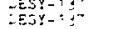 & 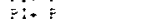 & $\because \because$ & & $\therefore \dot{r}-1+\infty$ \\
\hline$E-F$ & & $\cdot$ & & LESY_+ & $p: p$ & $-\cdots$ & & 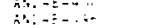 \\
\hline$\therefore-\quad$ & & $\because$ & & $\therefore E \therefore-D^{*}<t$ & 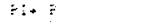 & $\cdot \ldots$ & & $\Leftrightarrow-\cdots$ \\
\hline$\therefore \div$ & & $\therefore \therefore$ & & $2 E 5 Y-4$ & $\bar{F} ;$ & $\ldots$ & & $\therefore \rightarrow=-2=$ \\
\hline$\therefore=$ & & $\Leftrightarrow$ & & CESQ-*: & $\mathrm{Fi}+\mathrm{F}$ & $\ldots$ & $\because$ & 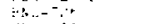 \\
\hline$\therefore-F$ & & $\therefore$. & & $\therefore L A:-E-3 X$ & $\because \because F$ & $\cdot \ldots$ & $\cdots$ & $\therefore+4 . .=3$ \\
\hline $\begin{array}{ll}5 \\
\vdots- & 5\end{array}$ & & $\because$ & $\because$ & CES:- : & i +5 & $\because \cdots$ & & $\cdots \dot{s}-\dot{y}-\dot{\gamma}$ \\
\hline $\begin{array}{l}\vdots- \\
\vdots-\end{array}$ & & $\because$ & & 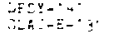 & 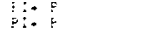 & $\because \cdots$ & & $-h-2=-3$ \\
\hline $\begin{array}{l}\vdots-\vdots \\
\therefore-5\end{array}$ & & $\ldots$ & & $\begin{array}{ll}-4 \\
A-1\end{array}$ & $\begin{array}{ll}F: F \\
P: * F\end{array}$ & $\therefore$ & & $-\dot{h}=-5=$ \\
\hline$E-i$ & & 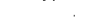 & & 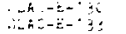 & $\because \vdots$ & $\therefore$ & & $\because h^{-}=-11$ \\
\hline$\therefore-\quad=\vdots$ & & & & $3 \hat{A}-5-3$ & 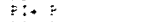 & $\cdots$ & & $\begin{array}{l}\because n-E-1 \geq Z A \\
\because n-E-C D E\end{array}$ \\
\hline$=-\therefore t:$ & & $\because$ & & $-5=5:-i 1:$ & $\vdots \vdots$ & $\ldots$ & & $\because$ IFA-nA- \\
\hline$\Sigma-:=2$ & & $\cdots$ & & SESY-" * & $8+5$ & $\ldots$ & & 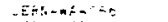 \\
\hline$=-\omega E$ & & $\cdot$ & & ESY - - & $\because F$ & c.. & 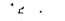 & rini- $-\therefore$ \\
\hline $5-\therefore$ & & $\because$ & & $\because=h=-E-s$ & $3: i$ & $\therefore$ & $\because \ldots-$ & $F: A_{L}-\because n$ \\
\hline$\therefore-5 i$ & & $\ddots$ & & ESY_-4 & $P:+i$ & $i \cdots$ & $\because \because \ldots$ & $\operatorname{tin} A-240$ \\
\hline $5-2 E d$ & & $\because \ldots$ & & $\because-2 E-E-\therefore C$ & $F: P$ & . $\ldots$ & & $\therefore E F+n-m i-\ldots s$ \\
\hline$E-2=2$ & & $\ldots$ & & $\therefore h \bar{E}-\vec{E}-3 C$ & $E+?$ & $+\ldots$ & & $B-S-n-2: 0$ \\
\hline$\therefore-t i E:$ & & , & $\cdots .8$ & $\therefore \angle C-E-121$ & 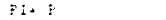 & $\because \therefore$ & & FNA:- -24 \\
\hline$E-A$ & & $\therefore$ & 7.5 & $\therefore \in L=E-121$ & $\div i+i$ & -7 & & $\because: 66_{2}-n^{2}$ \\
\hline$E-H E$ & & $\therefore$ & & $-E S Y-1 \alpha 1$ & $\mathrm{ri+F}$ & $\therefore$ & & FAAL- : IS \\
\hline$\dot{E}-5 \dot{E}$ & & $\therefore$ & & $275 Y-141$ & $\because 5$ & $\therefore \ldots$ & & $\because x_{i}-1$ ist \\
\hline 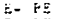 & & a. : & & SESY $=4$ & $P i+?$ & $\because \ldots$ & $c^{3}=2$ & $\therefore E k+k-\pi-0,6$ \\
\hline$E-\dot{E}$ & & $\because \therefore$ & & EESY- 4 & $P:+r$ & $\because 1$, & & $\therefore E$ I \\
\hline$E-5:$ & & $\therefore 3$ & & DEsY-4: & $P i+F$ & $5 \ldots$ & & ERK-Ih $-C^{2}$ \\
\hline$\therefore-4 i$ & & $=$. & & DESY-14 & $P: P$ & $\because \ldots$ & & EEki-nh-OCO \\
\hline$E-2 i$ & & $\therefore$ & & LESY-" & $5 !$ & $\therefore \therefore$ & & CEFN-mA-0 0 \\
\hline$E-5 i$ & & 7.3 & & DËSY-141 & FI. F & $\therefore$ & & $E S A L-324$ \\
\hline $3-10$ & C:EYS & $<3.2$ & & $\therefore A C-E-i 37$ & PI. & 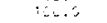 & & PKR--3o! \\
\hline$=-$ & & 5.2 & 20.0 & $\therefore E R N-W h-06+$ & PI.? & 12.6 & & F:On- $-0 A$ \\
\hline 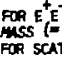 & ooniding & 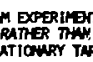 & $\begin{array}{l}\text { WE GIVE } \\
\text { ET. }\end{array}$ & TER-OF- & $\begin{array}{l}P_{1 *} \\
P_{1} * P \\
P \vdots+P \\
P I+P\end{array}$ & $\begin{array}{l}0.0 \\
10.0 \\
20.2 \\
20.2\end{array}$ & & $\begin{array}{l}\text { NA }--15 A \\
\text { FNA- }-350 \\
\text { FWAL- }\end{array}$ \\
\hline$E+E-$ & & ? & & SLAC-5P-025 & $\begin{array}{l}P I+P \\
P I+P\end{array}$ & $\begin{array}{l}: 00.5 \\
: 00.5\end{array}$ & $\begin{array}{l}230.4 \\
300.0\end{array}$ & 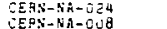 \\
\hline$\dot{B}+\bar{E}-$ & & ? & & $5 L A C-5 P-026$ & $3 I+P$ & 120.0 & 350. & FNAL-258 \\
\hline
\end{tabular}


BEAY-TARGET-MOMENTUM INDEX

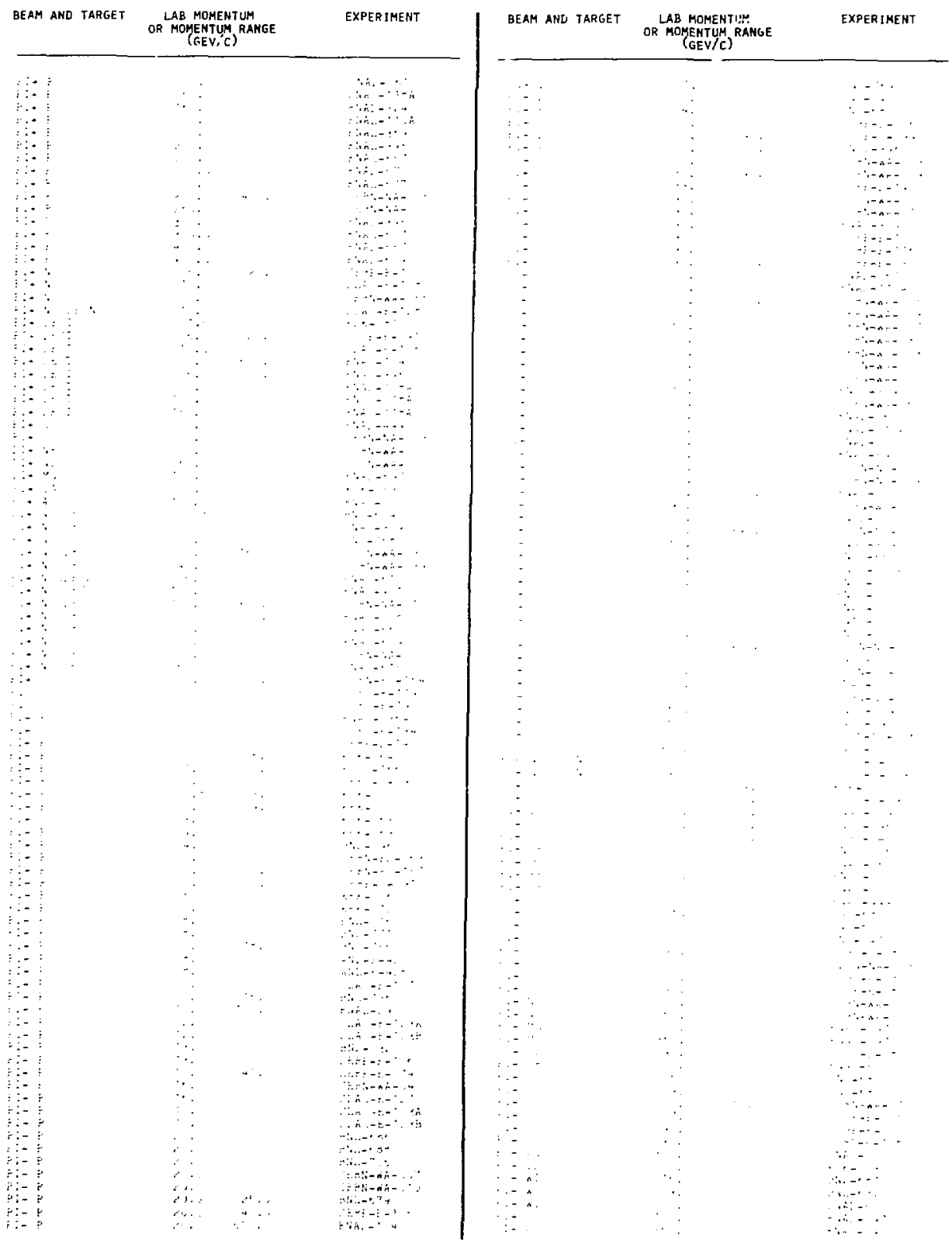



BEAM-TARGET-MOMENTUM JWDEX

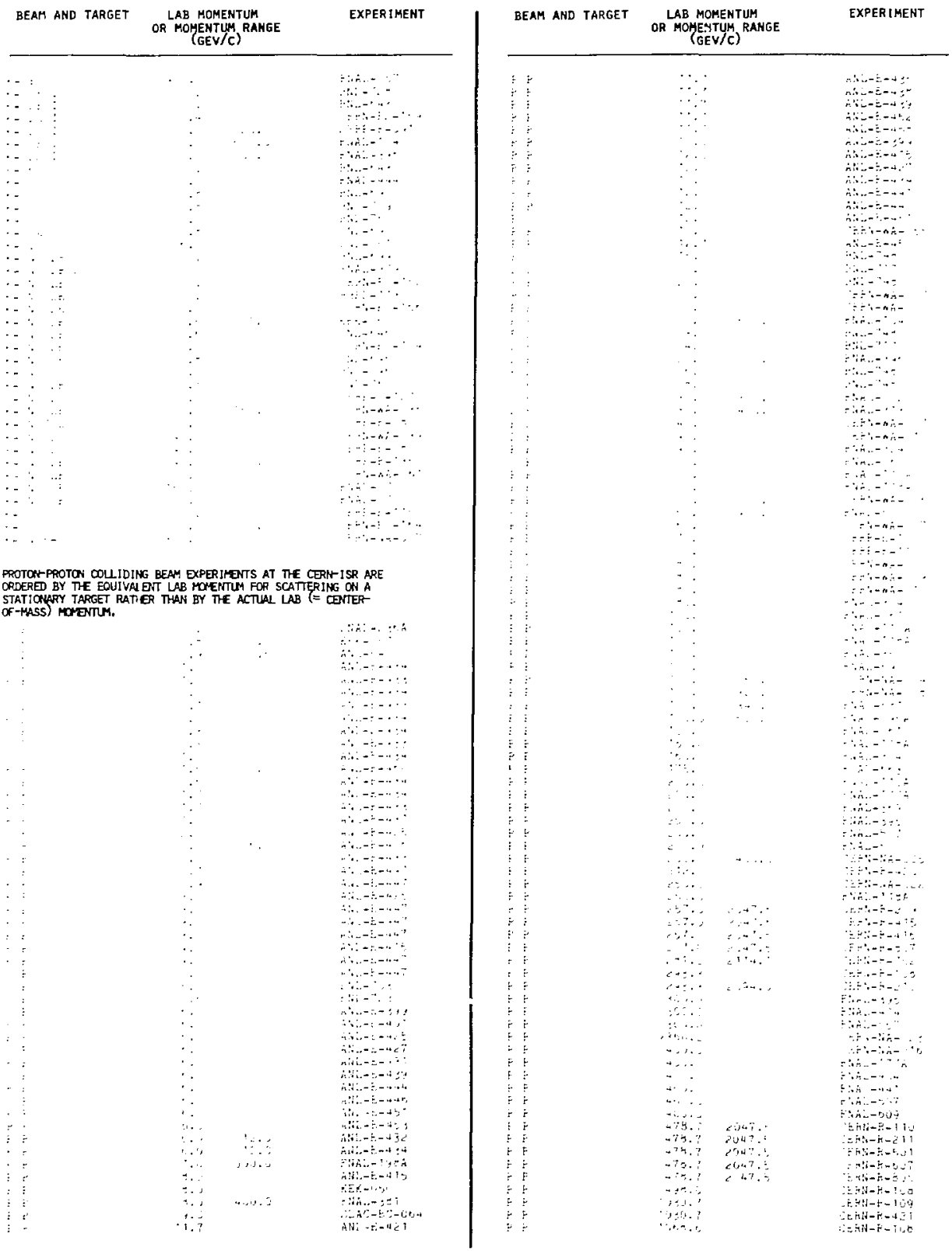


BEAM-TARGET-MOMENTUM INDEX

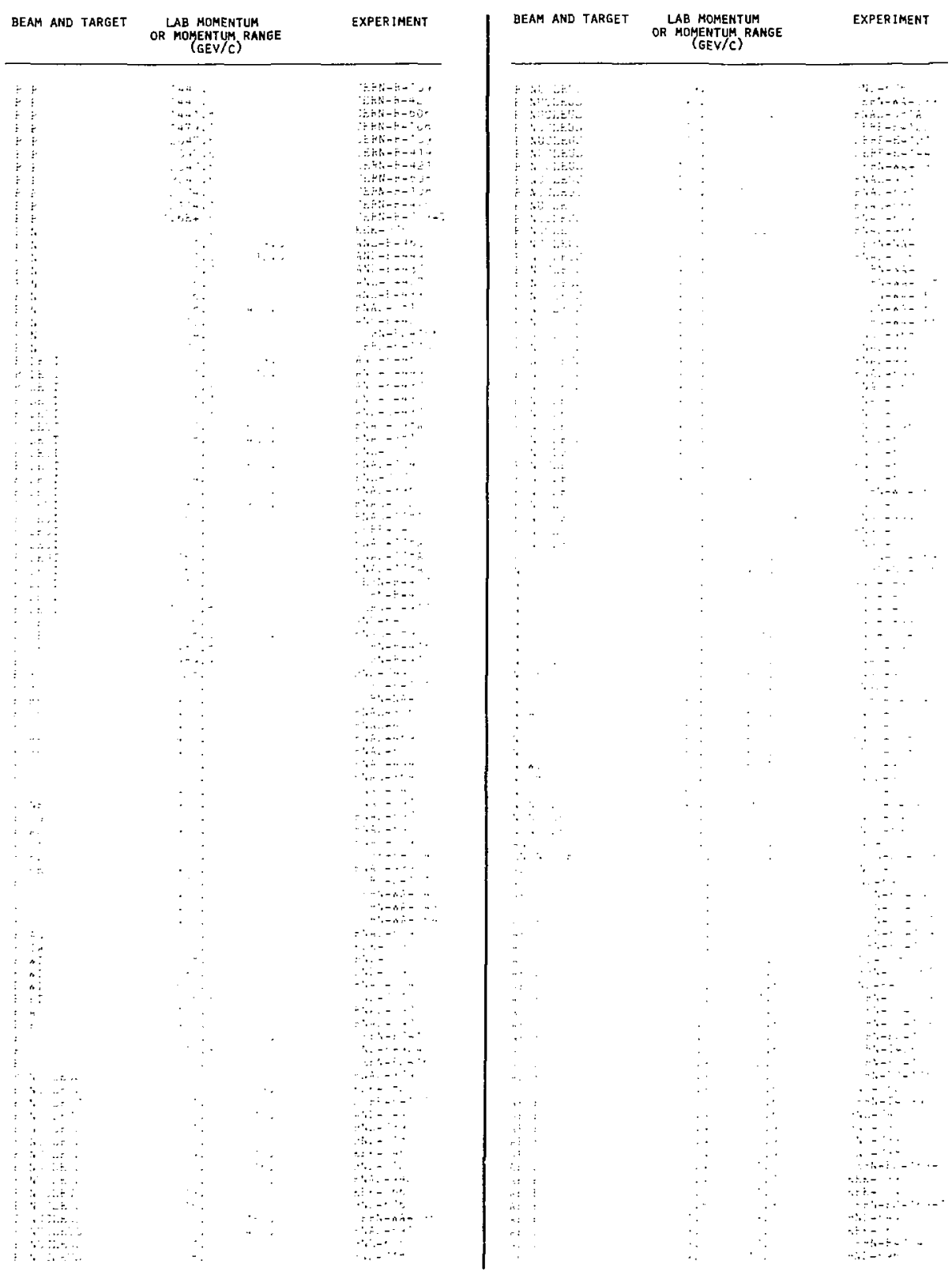


BEAY- JARGE I-MOMENTUM INDEX

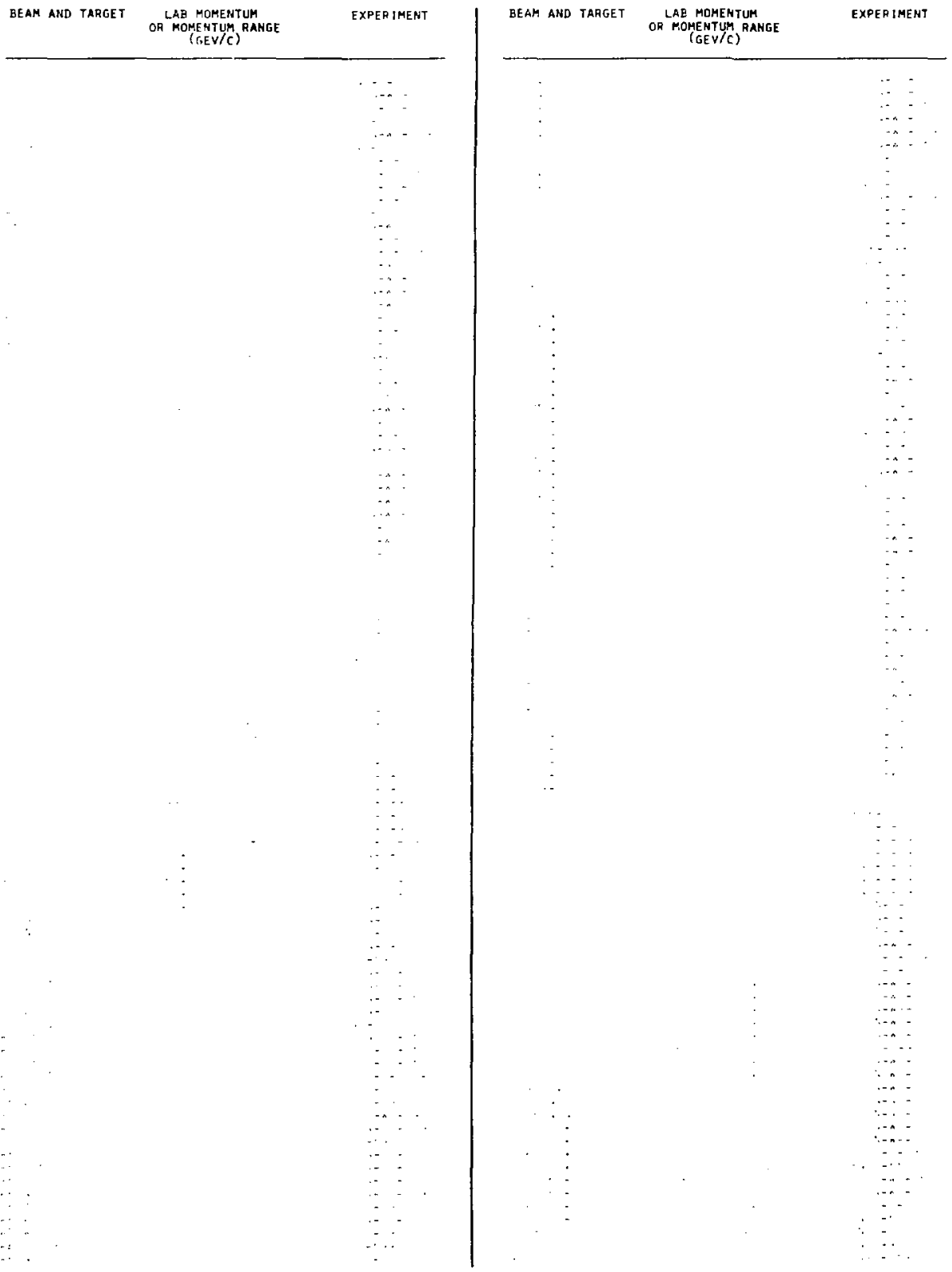


11

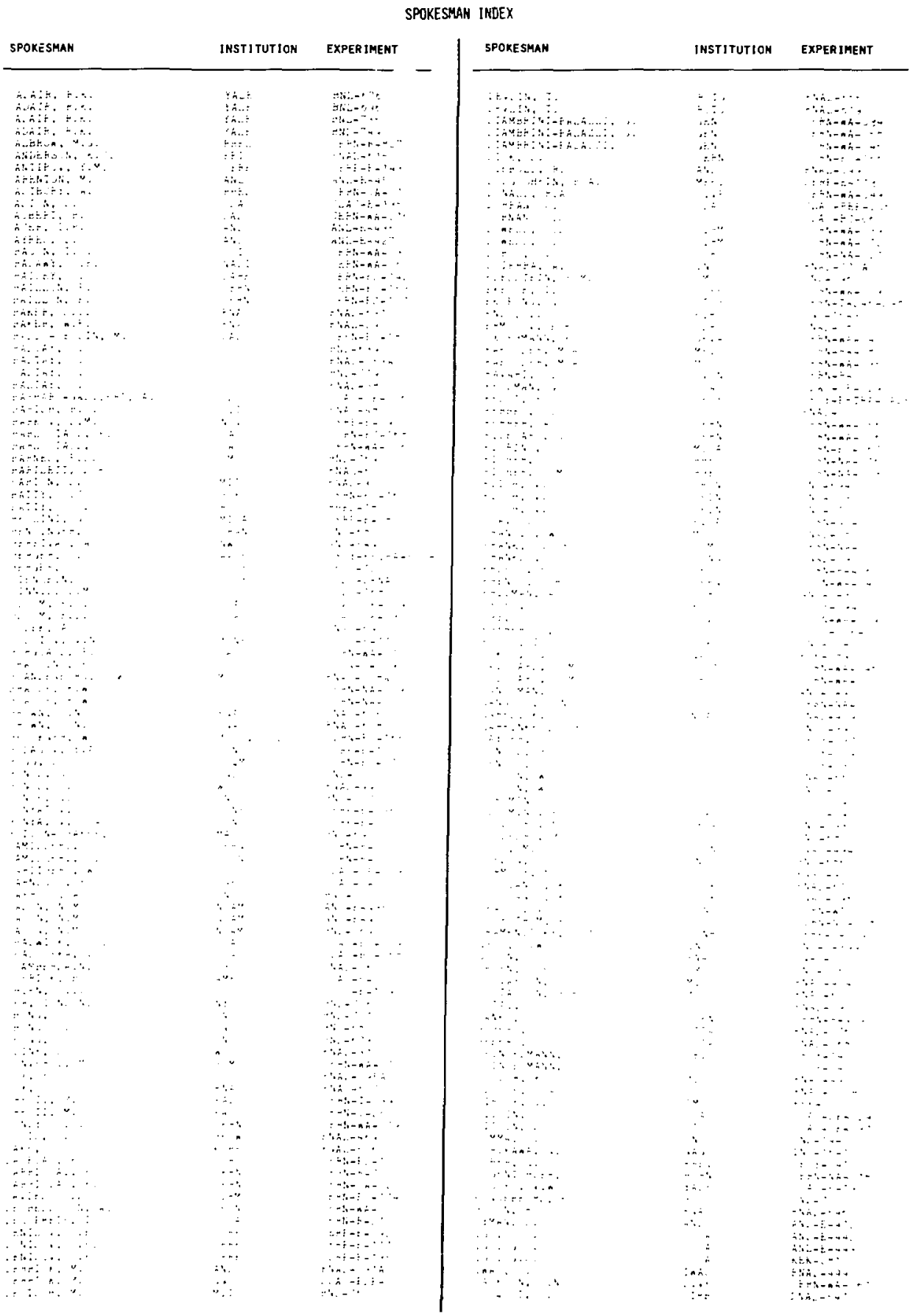


SPOKESHAH INDEX

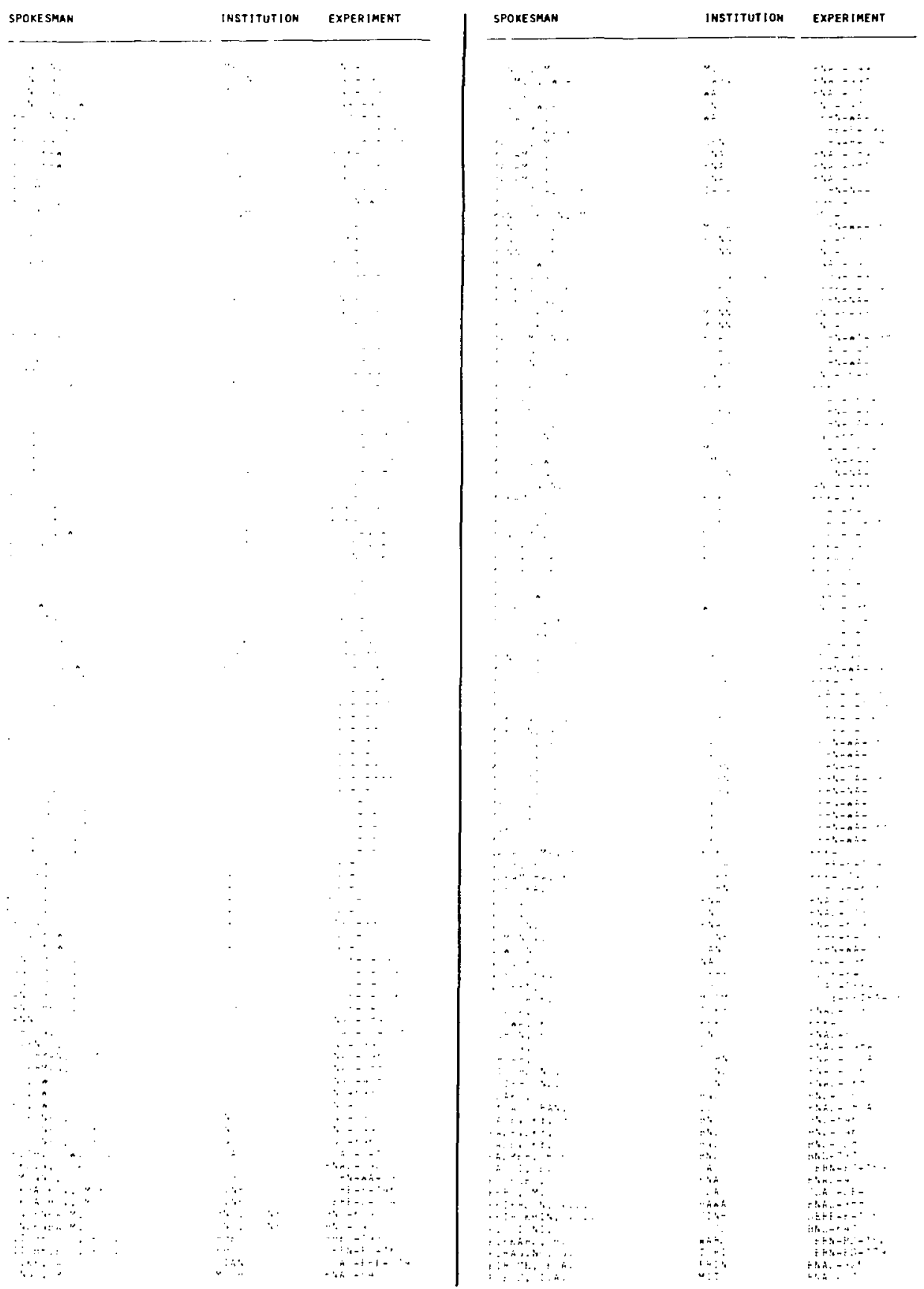


13

SPOKESMAN INDEXX

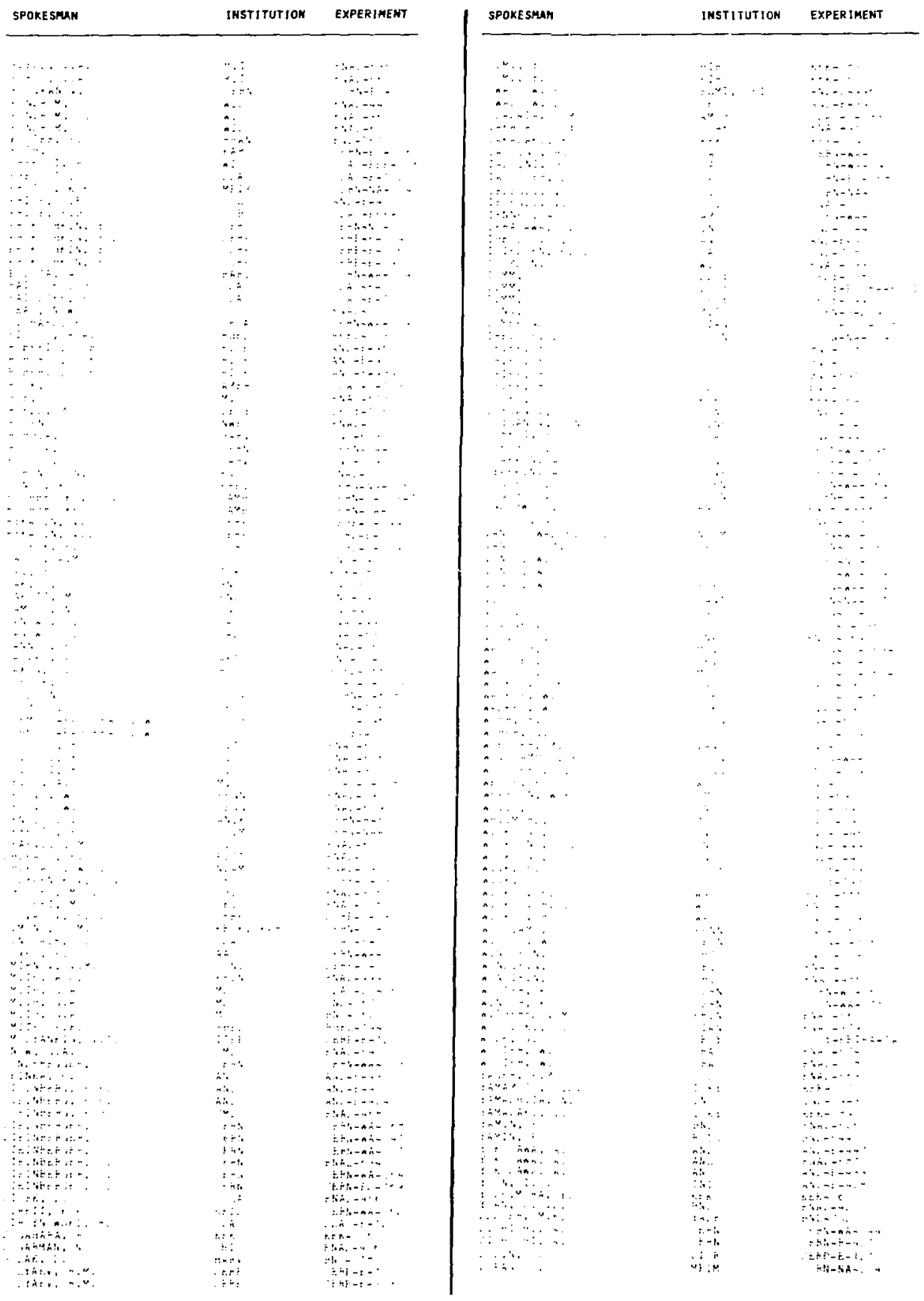


CEARAR/P CER

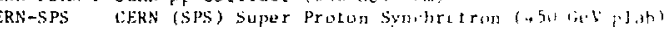

Hamburg Deuthes Elertron Symertictron ( 7 , S Giel phat

DESY-DORIS llamburg (Duris) filertrot-positron Ring allon liel arll

DESY-PETRA PETRA e e Colliuing Beatns (\$0 CoK erm)

FNAL FNAL batavia troton Synothrotron (5no GeV plab)

ENAL Batavia Praton Synchrotron ( 500 GeV piab)
LEK (Japan) Protun Synrhrotron (13 GeV plab)

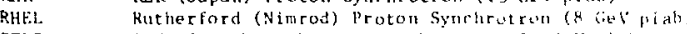

SERP IHEP Serpukhov proton Synrhrotron (ib liel what

SLAL Stantord telertron linear arcelerator (33 lic. phat

SLAC-PEP SLAC Posicron-Elertron P'rojert (J) (aeV erm)

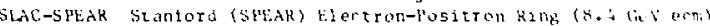

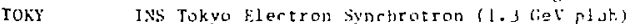

\section{DERECTOR VOCABLALAY}

For bubble rhambers we use a ronstrur tion surh as $\mathrm{DBC}-2 \mathrm{M}$

or

or

\section{HBC-1 SFT-HYB}

HL.B. $-\mathrm{BFBC}-\mathrm{TSS}$.

The first element, one ot

SBC
UBC

OBC

HEBC

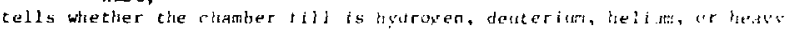

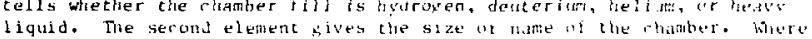

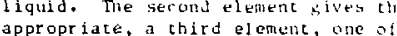

MYte,
RAP

RAP

indirates chat the rhamer is part of a hybrid systen, ar that it in ralit ryrliag, or that it colltains a trark-sensitive carget.

For non-bubble-rhamber decertors the abbreviacans are:

General:
CNTL

CNIK

EMUL

OSPK
OTHE.K ralorimeter

rounters (mo rhatibers)

rombinations of Jiffertut expes at dete.terh, ron

partirular one dumirant

emulsion or a detector like lexan where craks are

frozen in a soltd mediun

ol tiral spark chamber

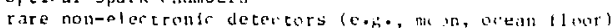

streanct chamber

trousilina radiation detertor

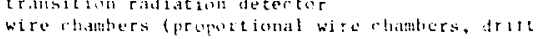

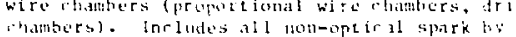

ionvelit wain

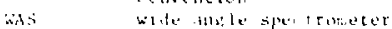

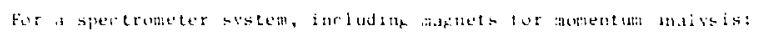

bas doinle arm spertrometer

Sins $\quad \therefore$ ingle artio spercroneter

SPIC: Leneril spertrometer systein not fitting one of the

wowe or where sperifir tope hut given

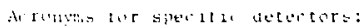

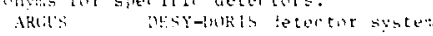

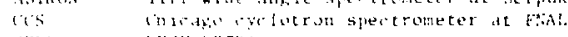

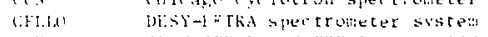

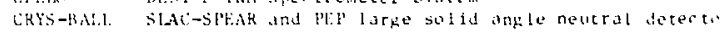

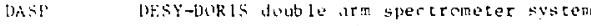

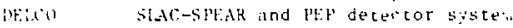

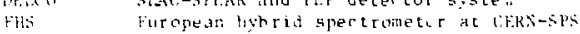

Whs awl effertive mass spertrometer

Fin fermalab multiparticle spererometer

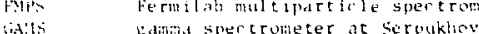

(iditis

H:'h

Rs

LA:

Lisis

thes

Yha:

Mikik-.

Makk -1

पhi'k $k$ k-

:Ir's

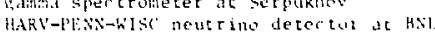

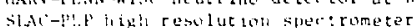

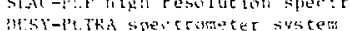

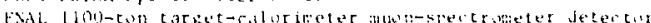
her neutriat pisysios

Slac larse aperture sulenoid spererometur

MSY-DNR Is decertar system

itsiY-l't TRA spectrometer suster:

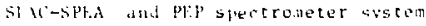

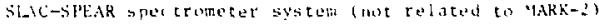

BSL mulcipartirle sperttometer

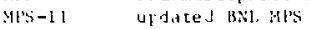

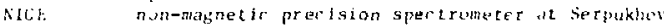

Nimetis

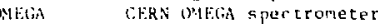

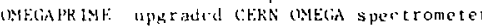

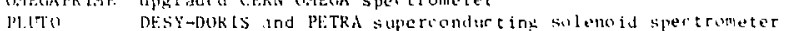

12:

SISF

St:

$\sin (\sin$

Sivichent

Sist

TAsist

THLAS

TUKI WA

Th'

DESY-DORLS and PETRA suncriondarting solemo id sper

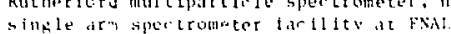

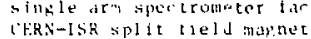

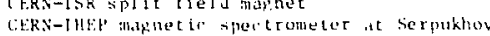

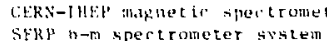

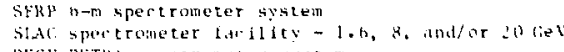

DESY-P'ETA spertrumeter systetil

KkK tarket-ombodicd targe-aperture shetroneter

KFK spertrometer

SLAC-PEF t ine projeltion "hamber

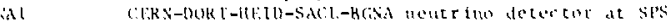

$\therefore-4.19: 1 \mathrm{~s}$

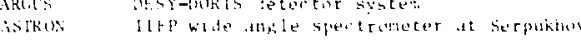

Slat:-Plt" this!netir calorimeser 


\section{REACTION DATA DESCRIPTOR VOCABULARY}

The deta descriptors reier to the nature of the data to be taken in an experimant. Any of the variables below can also be understoud to refer to functions (including averages or other monents, but not derivatives or lategrals) of that variable, unless such tunctions involve other variables from the liat. For date which are to be presented as a func:ion of two variables, wuch as a scatter plot, combinations such as MASSMASS are used.

\section{GEHERAL}

Gross section, cross section ratio, and eross sect1o it upper 1 imit. Can aluo be listed for very rare reactions whose existence is being establighed, even though the number of events has not been

converted to cross aection. Does not include paranetrizations of the croes section, e.g., as a function of energy.

AHGP Production angular diatribution, 1.e., of one or more of the outgoing particles relative to one of the incident particles. Includes $\mathrm{do} / \mathrm{dn}, \mathrm{d \sigma} / \mathrm{dt}, \mathrm{do} / \mathrm{dt}^{*}, \mathrm{do} / \mathrm{dQ}^{2}$. etc. Also the equivalent, expressed as moments or polynomial expansion coefficients. Also Invariant cross section as a funition of production angle or $t$. By convention, does not include rapidity or its epproxiantion, $y=-\ell n \tan \theta / 2$ (see P). Includeg linpacr par anaters and slopes of $\mathrm{d} \sigma / \mathrm{dt}$.

agular diotribution between or among particles in the final state. Includes al to angular distribution involving decay product of particles linted in the reaction, even though those decay produrts are not themselves explicitly listed. Includes ans]as used to the the decey of a though the coordinate system axes may be defined witli respect to the incldent particles (e.g., Jackson angles, etc.) Also the equivalent, expreased a moments, etc.

Mass. Mare epectrum, naso ${ }^{2}$ epectrum, or invariant cross 5e'tion as a function of mass or mass squered.
Transverse momentum $\left(P_{T}\right)$ spectrum, $P_{T}^{2}$ spectrun, or 1.variant cross section as a function of $\mathrm{p}_{\mathrm{T}^{*}}$ Does not include momentum transfer epectrum (eee ANGP). Includea transverse mass $-\left(p_{T}^{2} m^{2}\right)^{1 / 2}$, unless the particle mass (m) is also variable.

\section{REACTION DATA DESCRIFTOR VOCABULARY (CONT'D)}

Any function of outgolng momentum or energy not included in any of the above. Includes, $E_{2}$ y (rapldity, also rapldity gaps), $x\left(=\mathrm{P}_{\| \mid}^{*} / \mathrm{p}_{\max }^{*}\right), \mathrm{P}_{\|}$, or other modentum or enargy variable.

(for proposals unly) Exper1ment propoces to messure complete our-vectors, wi thout specifying exactly wat analyois of thes will be done.

\section{AMPLITUDES}

Functions linear in the amplitudes (1.e., Involving the phases). Partial-wave amplitudes. Includes formation partial waves and production partial waves. Any attempt to measure asplitudes of definite 1 (angular momentum). Includes scatering length and ef fective range.

$\mathrm{AM}^{-}$Amplitude not jecomposed into states of definite 1, RR/CM ratio, helicity amplitude, etc.

\section{VARIABLES RELATED -TO SPIH}

Density matrix elementa, including joint density matrix elements.

Final state spin-1/2 polarization measurement. Includes Wolfenstein spin rotation parameters. Includes measurment of asymetry of a polarized target when $1 \mathrm{t}$ is equal to the fianl state polarization.

Asymmetry in scuttering off a polarized target and/or with a polarized beam (wlth exception of special case noted under POL).

\section{MULTIPLICITIES}

HULT Kultiplicity digtribution, its average, ratio, or monents. Generally used in association with final states of the form N(PRONC), N(HADRON), etc., so that the Individual final atates are usually not listed. 


\section{KIAEMATIC VARTABLE VOCABULARY}

The Beam "momentum" designation given in parentheses following the numerical value and units can be one of the following:
PLAB
ELAB bean tnergy in the lab frame.
TLAB beam kinetic energy in the lab frane.

total energy in the CM frame.

5 total CM energy squared.

For colliding beam experiments, the momentum of the second beam 15 given indented below that of the first. Alternatively, a single line with the total center-of-mass energy or equivalent lab bean momentum may be given

For electroproduction or other reactions involving a virtual photon, the second and third lines indented below the beam monentum specify the equivalent of the mass and monentum of the virtual photon. These can have the following designations:

H nass of the target-virtual photon system.

H2 square of $H$.

Q2 absolute value of the mass squared of the v1rtual photon absolute value of the squared 4-momentum transfer to the electron.

NU energy of the virtual photon in the lab frame = energy loss of the electron to the lab frame.

\section{PARTICLE PROPERTY DESCRIPTUR VOCABULARY}

The foliowing descriptors are used to designate variuus types of particle property deta:

MASS Mass or mass difference.

W Total width, cotal rate, mean life. Also differences and ratios of these.

PH Partial width, partial rate, as well as any racio or product of these such as branching ratio ur integrated cross section. Also upper limits on these. Also differences of these unless included in DEC (DEC+inc dudes charge asymmetry $\delta$ for $K_{L}+\pi l v, \eta$ for $K_{L}+\pi+, x+1 y$ for $\left.\mathrm{K}_{\mathrm{S}} \rightarrow \pi^{+} \pi^{-} \pi^{0}\right)$.

MoM Electric moment, magnetic mument, charge radius, motient ratios.

DEC Neak or electromagnetic decay parameter as defined by Raview of Particle Properties, Rev. of Mod. Physics 52, No. 2, Part II, Ap: 11 1980 , Sec. VI. slopes $g$ and slope difference $u(C P$ Tiol) $\quad$ for $K \rightarrow 3 \pi$

form factors $E_{+}, E_{-}, f_{0}, \lambda_{+}, \lambda_{-}, \lambda_{0}, E_{,} E_{S}, f_{T}$ for $\mathrm{K} \rightarrow \pi l v$

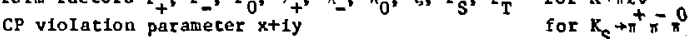

charge asymmetry $\delta$

CP viol. parameters $n_{+}, \eta_{00}, \phi_{+}, \phi_{00}, c, \varepsilon^{*}$

$\Delta S \neq \Delta Q$ parameter $\mathrm{x}$

charge asymmetry

$\left|g_{\mathrm{A}} / \mathrm{g}_{\mathrm{V}}\right|, \delta, a, B, \gamma, \phi, \Delta$

for $K_{L}+\pi l v$

for $x \rightarrow \pi$

for $x^{d}+\pi^{+} x$

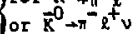

for $n$ decay

for baryon decay

QN Quant un numbers.

EX Existence (e.8., particle scarch result, even if negative, or evidence for presence in a mass spectrun). 
AACH Phys. Inst. der Tech. Hochschule

AARH Aarhus Unty.

AAAD Abadan Insc. Of Technology

AICH Aichi Educational Univ.

AIKo Inst. Kernphysics Onderzoek

AKIT Akita Univ.

AlsA State UnIv. of the York at Albany

AMA Kazakh Inat. Sor High Energy Physics

ARR American Univ.

MES Anes Lab

MIST Univ. of Amsterdam

ANIK Materder

ANIK Amaterdem Mikhef

ANKA Middle East Techn

ANL Argonne Nat. Lab.

ATEN Nuclear Res. Centre Lamokritos

AMRE Atonic teapons Research Establishment

BARI Un 1v. di Bar1

BASL Basle Univ.

AEDF Bedford College

BEIJ Beijing Univ.

BELG Inot. Interuniv, des Sc1. Nuclear

BERG Fyaisk Institut

BERL Inst. Hochenergiephys. DAW

DERS Unty. Bern

BGA Univ. di Bologna

BHE Inst. Of High Energy Fhyeics

BIEL Univ. Bielefeld

DIRK Birkbeck College

DIRH Birainghan Univ.

DNL Brookhaven National Lab.

BOHR Niels bohr Insticute

Bars Bombay Univ.

Bors Univ, Bonn

bOST Boston Univ.

BRAR Brandels Univ.

BrCo British Columbia Univ.

BRIS H. H. W1lls Phys, Lab., U. of Bristol

DROW Brom Univ.

BRUX Univ. Libre de Bruxelles

BrL Bell TeI ephone Labs.

BUDA Central Research lnstitute of Physics

BUTF State Univ. of New York at Buffalo

CaMp Cambridge Univ.

CAMD Cambridge Univ.
CALC Univ, of Calcutta

CALC Univ, of Calcute

CARL Carlton Univ

CAvE Cavendiah Lab., Canbridge Untv.

CDEF College de France

CERN European Org. for Nuclear Research
Aachen, Germany

Aarhus, Denmark

Abadan, Ir an

Toyota, Aich1 Pref., Japan

Amsterdam, Netherlands

Ak1 ta, Japan

Albany, NY, USA

Alma-Ata, USSR

Washington, DC, US I

Ames, Iowa, USA

Amsrerdas, Netherlands

Amsterdam, Netherlands

Ankara, Turkey

Argonne, I11., USA

Tucson, Ar1z., USA

Arhens, Greece

Alder tagton, England

Bar1, Italy

Basle, Switzerland

London, England

Be1j1ng, China

Bruxelles, Belgtum

Bergen, Norway

Zeuthen/Berlin, UDR

Zeuthen/Berlin,

Bologna, Italy

Belj1ng. China

Bielefeld, Gerni

Lirmingham. Eng tand

B1rmingham. Eng tand
Upton, L. I., NY. USA

Upton, L. I. "NY. USA

Bombay, Ind1a

Boan, Gertmany

Boston, Mass., USA

Haltham, Mass, USA

Vancouver, Canada

Bristo $i$, England

Providence, RI, USA

Bruxelles, Belgium

Murray Hill, NJ, USA

Budapest, Hungary

Buffalo, NY, USA

Cambridge, England

Calcutta, Indl

Ot tawa, Canada

Cleveland, Oh1o, USA

Cambridge, Eng land

Par1s, France

Genevn, Swltzerland
CHIC Univ. of Chicaro

CINC Unte of Cincinnati

iIT Calif, Institute of Technology

CLFR Univ. de Clemont-Ferrand

GiU Carnegle-Mellon Univ.

Colo Univ of Colorado

COLU Colunbia Univ.

COPE Copenhager Univ.

CORN Cornell Univ.

CKAC Inst, for Nuclear Research

CUNY City Univ, of New York

CURI Plerre et Mirie Curle Univ., Paris VI

DARE varesbury Nuclear Physics lab.

DESY Deutsches Elekt ronen-Synch.

DOE Department of Energy

DORT Univ. Dortmund

DUkE Duke Univ.

DUUC University college

EDIN Univ. of Edinburgh

EF I Enrico Fermi Inst. for Nuclear Studie

ELMT Elmhurst College

EPOL Ecole Polytechnique

ERLA Univ. Erlangen

ETHZ Swlss Federal Inst, of Technology

FIRZ Univ, di Firenze

FNAL Ferm1 National Accelerator Lab.

ERAS Lab. Nazional1 del S1ncrotrone

FREI Univ. Freiburg

FSU Florida State Univ.

GENO Untv, di Genova

GESC General Electric $R$ and $D$ Center

GEVA Univ. de Geneve

GIT Georgia Inst. Tech.

GLAS Untv of Glasgow

GAS George Mason Univ.

GUIL Univ. of Surtey at Gull ford

HA IF Technion - Iarael Inst. of Technology

HAM? Un 1v. Hamburg

HRV Harvard Univ.

Univ of Hawa 11

HELS Helsing in Yllopisto

HIRO Lli roshima Un1v.

HoUS Univ, of houston

HRSK H1rosaki Univ.

IIT Illinols Inst. of Tech.

ILL Unive of Illinols

ILLC Univ. of Illinols at Chicago

Univ. of Indiana

INNS Innsbruck Univ.
Chicago, Ill, USA

CIncinnati, Ohio, USA

Pasadena, Calif., USA

Clemont-Ferrand, Franc

Pittsburgh, PA, USA

Boulder, Colo", USA

New York, NY, USA

Copenhagen, Denmark

Ithaca, NY, USA

Cracow, Polend

New York, NY, USA

Paris, France

Darestury, England

Hamburg, Germany

Heshington D.C., USA

Dortuind - Hornbruch, Germany

Durhsin, MC, USA

Dublin, Ireland

Edinburgh, Scotland

Ch1cago, Ill., USA

Elmhurst, Ill., USA

Palalgeau, France

Erlengen, Gerruany

Zur 1ch, Sw1tzerland

Firenze, Italy

Batav1a, Ill., USA

Frascati, Italy

Fre1burg, Germany

Tallahassee, Fla., USA

Genova, Italy

Schenectady, NY, USA

Geneva, Swltzerland

Atlanta, Georgia, USA

Glasgow, Scotland

Falrfax, VA, USA

Ga1l ford, Surrey, England

Halfa, Israal

Hambur 8 , Cermany

Cambridge, Mass,, USA

Ho nolull u, Havaif, USA

Heidelberg, Germany

Helsink1, Finland

H1rosh1ma, Japan

Houston, Texas, USA

H1 rosak1, Japan

Ch1cago, IIl., USA

Urbana, IIl, USA

Ch1cistu, Il1., USA

Bloonington, Ind., US
Innebruck, Austria 


\section{INSTTTUTION VOCABULARY (CONT"D)}

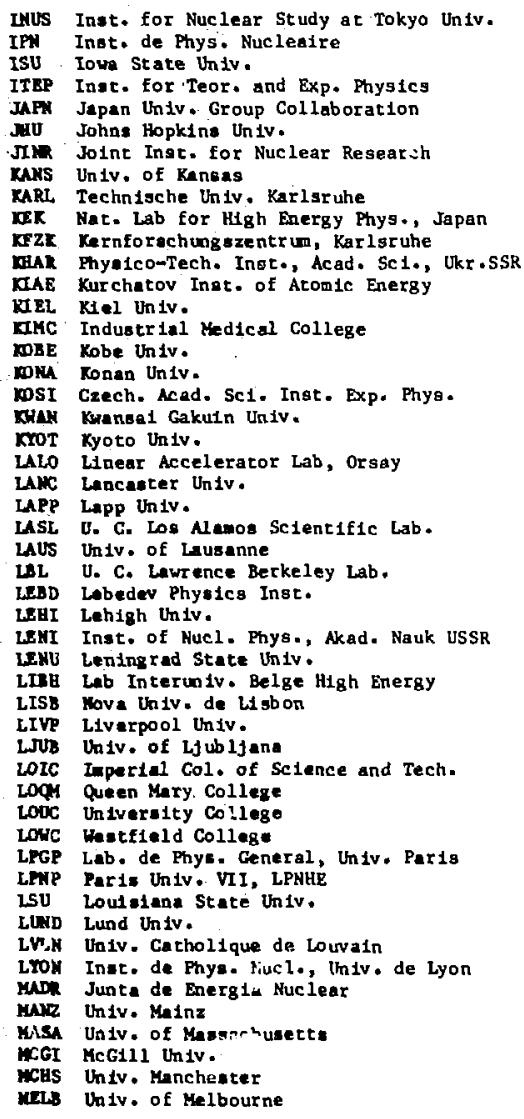

\section{INSTITUTION VOCABULARY (CONT"D)}

Tokyo, Japan Orsay, France mes, Iowa, USA Moseow, USSR

apan

altimore, Md., USA Dubna, USSR

lawrence, KBnsas, USA

karlsruhe, Germany

Tsukuba-gun, Japan

Leopoldshaven, Germany

HoScOv, USSR

K1 takyushu, Japan

Kobe, Japan

Kabe, Japan

Hyogo-ken, Japan

Kyoto, Japan

Kyoto, Japan

Lancaster. Fing land

Annecy, France

Los Alamos, NM, USA

Laualanne, Switzerlan

Berkeley, Calif., USA

Moscow, USSR

Bethiehem, PA, USA

Leningrad, USSR

Leningrad, USSR

Brusse1s, Belglum

Lisbon, Portugal

Liverpool, England

Lfubljana, Yugoslavia

London, Eng land

London, England

Lond on, England

London, England

Pandon, England

Par1s, France

Baron Rouge, LA, USA

Baton Rouge,

Louvaln-la-Neure, Belg.

Villeurbanne, France

Madrid, Spain

Mzinz, Germany

Anherat, Mass., USA

Montraal, Canada

Manchester, England

Parkville, Australla
MEXU Univ. Nal: Auconona de Mexico

MLAM Mian 1 Univ.

MICH Undv, of Michigan

MILA Univ. di Milano

MIHN Univ, of Minnesore

MINR Insticure for Nuclenr Research

MIT Massachusetts Inst. of Technology

MONS Univ. de 1 'Etat, Mon

MosU Moscow State Univ. Inst. of Nucl. Phys.

MPE I Noscow Phys. Eng. Inst.

MPIH Max-Planck-Inst, fur Phys.-Astrophys

MPIM Max-Planck-Inst. fur Phys,-Astrophys.

MSU Michigan State Univ.

MTHO Mt. Holyoke Co! lege

MUN I Munich Uniy.

MURA Midwestern Univ. Research Assoc.

MADI Mohamed El-Nadi Research Center

NAGO Nagoya Univ.

MANC Univ, de Nancy

MAPL Univ. di Napoli

MARA Nara Women's Univ.

MARU Nara Unty.

NCCI North Central College

NDAM Univ, of Notre Dame

NEAS :ortheastern Undv.

NEUC Univ, of Neuchatel

NEVI Nev1s Lab.

NIIG NIIgata Univ.

NItUN Nihon Univ.

NIJM R. K. Un iv, $1 / 2$ Jmegen

NILU Northern Illinois Univ.

NORD Nordisk Ins, for Teor. Atomfys.

Novo Inst. of Nuclear Physics

NRL Naval Research Laboratory

NRLO Naval Research Lab

NSF National Science Foundacion

NTUA National Technical Univ, of Athens

NWES Northwestern Univ.

NYU New York Un iv.

CakM Jakland Univ.

OKAY Okayama Un Iv

OPEN Open UnIV.

cEn!. Ock Ridge Naclonal Lab.

ORSA briv. de Par1s, Fac. des Science

OSAK Osaka Un1V.

OSKC Osaka Gicy Univ.

OSLO OSlo Undv.

OSSE Science Educ. Inst. of Osaka Pref.

osu oh to State Univ.

OTTA UnIv, of Ottawa
Mexico Cicy, Mexico

Hian1, FL, USA

Ann Arbor, Mich., USA

Ann Arbor, Mich

MInneapolis, MInn,, USA

Moscow, USSR

Cambridge, Hass., USA

Mons, Belgiu

Moscow, USSR

Mosc OW, USSR

Heidelberg, Cemany

Munich, Gerany

East Lansing, Hich., USA

South Hadley, Mass., USA

Munich, Gerwany

Stroughton, WI, USA

Cairo, Egypt

Nagoya, Japan

Nancy,

Napoli, Ital

Nara, Japan

Nara, Japan

Naperville, Il, USA

Norre Dank, Ind., usA

Boston, Hass., USA

Neuchatel, syitzerland

Irvington-on-Hudson, HY, USA

N1i: ata, Japan

Tokyo, Japan

Mjmegen, Wetherlands

Dekalb, Il l., USA

Copenhagen, Dermark

Thehtenton, D.C USA

Mashing con, D.C., USA

Orlando, TL, USA

hashing ton, D. C. , USA

Athens, Greece

Evaneton, Ill., USA

New York, NY, USA

Oakland, Mich., USA

Okayama, Japan

Milton Keymes, England

Oak RIdge, Tenn., USA

Orsay, France

Oraka, Jupan

Osake, Jupan

Oelo, Norway

Osake, Japan

Colmbus, Oh1o, USA

Ot tawa, Cenada. 


\section{INSTITUTION VOCABULARY (CONI'D)}

OXF Oxford Univ,

OXF Oxford Univ.

Pavi Univ. di Pavia

PEWh Untv of Pennsylvania

PISA Untu. di PIsa

PITT Univ. of Pittsburgh

FRAG Institute of Physics, CSAV

PRIM Princeton Univ.

PURD Purdue Untv.

REHO Weirmenn Inst. of Sclence

RHEL Rutherford High Energy Lab.

RICE William Mersh Rice Univ.

ROCH Univ. of Rochester

Rock Rockefeller Univ.

ROMA Unive di Roms
RUTG Rutgers Univ.

SAGL Center d'Etudes Nuclear Saclay

SAGA Center diniv.

SAIT Sactame Univ.

SNivT Untv, de Santander

scuc Uive of South Carolifa at Columbia

SEOU Korea Univ. at Seoul

SERP Inst. of High Energy Physics

SHEF Univ, Of Sheffield

SHIS ShInshu UnIv.

Stir Univ, of Southampton

SIEG Siegen Unitv.

SIDY Simens Schuckertwerke AG

SIAC Stanford Linear Accel. Center

SIAS Southeastern Magaachusetts Univ.

SOFC High Inst. of Chen. Tech.

SOR I Bulgarian Acad. of Science

SRIP State Res. Ingt. Photochem. Proj.

STAN Stantord Univ.

STEV Stevens Inst. of Tech.

STEV Stevens Inst of
STOH Stockholm Univ.

STOH Stockholm Univ.

STON State UnIv. of New York at Stonybrook

STRB Centre des Res. Nucleaires

SUFF . Suffolk Univ.

SYDN Univ. of Sydney

SYRA Syracuge Univ.

TATA Tata Inot. of Fundamental Research

TBIl Inst. of Phys., Acad. Science

TBSU Tbilisi State Univ.

TELA Un1v. of Tel-Av1y

TEAP Tample Univ.

TEM Un1v, of Tennegsee

THES Univ, of Thessalonik1

MSK Nucl. Phys. Inst., Tomgk Polytech, Inst.

MU Tokyo Metropolitan Univ.
Par1s, France

Philadelphia, PA, US.

Plsa, Italy

P1ttgburgh, PA, USA

Prague, Czechoslovak1a

Princeton, NJ, USA

Lafaye tre, Ind., USA

Rehovoth, Is rael

Chilton, Did., Oxon., Eng laud

Houston, Texas, USA

Rochester, NY, USA

New York, NY, USA

Roma, It aly

New Brunswlck, NJ, USA

Gif-sur-Yvette, France

Saga, Japan

Sa1tama, Japan

Santander, Spa1n

Columbia, SC, USA

Seoul, S. Korea

Serpukov, USSR

Sheffield, Yorks., England

Uatsumoto, Japan

Southampton, England

Huttental, Germany

Erlangen, rermany

Stanford, Cal1f., USA

North Dartmuuth, Mass., USA

Sof1a, Bulgaria

Sof1a, Bulgar 1

Moscon,

Stanford, Cal1E., USA

Hoboken, NJ, USA

Stockholm, Swe den

Stonyb rook, LI, NY, USA

Strasbourg, Franice

Suffolk, Eng land

Sydney, Australia

Syracuse, NY: USA

Bombay, India

TO11 1s1, USSR

Tel-Aviv, Is

Philadelph1s, PA, USA

Philadelph1a, PA, USA

Knoxville, Tenn., USA

Thessalonik1, Greece

Tokyo, Japan
INSTITUTLON VOGABULARY (CONT'D)

TNTO Un 1v. of Toronte

TOCA Tohoku-Gakuin Univ.

Toronco, Caneda

Xjyag 1, Japan

Se:da1, Japan

Tokyo, Jepan

Torino, Italy

Vancowver, Canade

Trieste, Italy

Toarak1, Japan

Medford, Mase., USA

Tokyo, Japan

Athen $\theta$, Greece

Belgrade, Yugoslavia

Berkeley, Galif., USA

Dav1 s, Calif., USA

IrvIne, Callf, USA

Los Angeles, callf., USA

Riverside, Callf., USA

Senta Barbara, Gallf., USA

Santa cruz, Callf=, USA

La Jolla, Callf., USA

Madrid, Spain

College Park, SD, USA

Albuquerque, New Hex., US

Monterey, Callf, USA

Salt lake City, Utah, USA

Utreche, Ne therlands

Utsumoraya, Japan

Uppsala, Sueden

Valencia, Spa in

Nashville, Tenn., USA

Petersburg, VA, USA

Poughke eps1e, UY, USA

Victorla, BC, seneda

Vienna, Austria

Charlot tesville, VA, USA

BlacksburB, VA, USA

Blacksb urB, VA,

Segttle, Wash., USA

vienna, tustrie

W11llamshurg, VA, USA

Wargaw, Poland

Madison, Hac., USA

Wuppertal, Germany

Hur zburg, Geruany

La rante, Hyoning, USA

Hew Haven, Conn., USA

Yereval, Armenta, USSR

Yokoham.1, Japan

nowsivilew, ont., Caneda

zurich, Switzeriand

YERE Yerevan Physics lnst.

Yoko Yokohama National Univ.

YORK York Universtty 


\section{PARTICLE YOCABULARY}

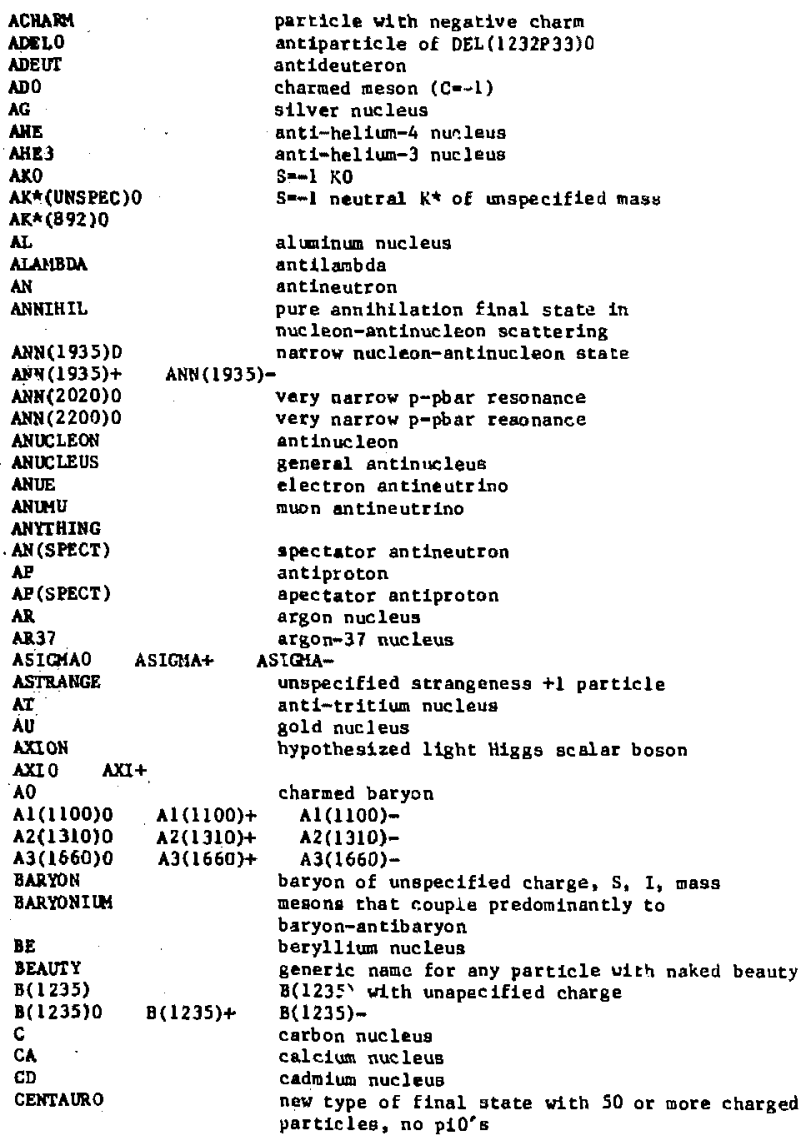

\section{PARTICLE VOCABULARY (CONT'D)}

CHARGED

CHARGED+

CHARIST-

CHARM

GHARMED-BARYON

CHI (UNS PEC)

CHI (UNSPEC)O

CHI (3510)

CHI $(3550)$

$\mathrm{CR}$

C12

C* (4.44)

DD a charged track originating from the prinary Interaction

positive charged particle

negative charged particle

charmed particle

charmed baryon of unspecified $C, S$, I, or charge unspecifled radiative decay product of pai (3700) unspecified radiative decay product of pai(3700)

radiative decay of psi(3700)

radlative decay of pgi( 3700$)$

chromium nucleus

copper nucleus

carbon-1 2 nucleus

4.44 kev excited state of carbon

diffraction dissoctation. To be followed by nanes of particles thich were somproduced,

$e+g$. DD $<P$ PIO>
DELTA 980$) 0 \quad$ DELTA(980)+ DELTA(980)-

DELO DEL(1 232P33)0

DEL+ LE? (1232P33)+

DE: + rAL(1232P33)+

De. DEL(1232P33)

DE'. UNSPEC) 0

LEL, (UNSPEC)+H

DEUT

DIBARYON

DIBARYON

Do

D+

$\mathrm{D} *(2010)+$

$D^{*}(2010)-$

D(UMSPEC)

EPSILON (700)

ETS

ETAPR IME

ETAPR LME/C

ETA/C

ETA(! 7 80)

EXOTIC-IESON

EXOTIS-NUCLEON

E+

$E+S$
$E+(S)$
$E-$

$E+(S)$
$E-$
$E-S$
$E-(S)$

$E \rightarrow(S)$

$E ; 1420)$

FE

$I=3 / 2$ baryon of unspectfied mass

I-3/2 baryon of unspecified mass

deutaron

$s=0$ dibaryor reconance of unopecteifed mass

$S=-2$ dihyperon resonance of unspacified nass

charned meson

charmed meson

charmed meron

charmed meson

charaed meson

then

p1-p1 S-wave (near $700 \mathrm{MeV}$ )

recurrence of ETA/C

$\mathrm{JP}=0$ - charmonlum state

cannot be formed of quark-antiquark

cannot be formed af 999

positron

two or more positrons

one or more positrona

electron

two or more electrons

one or more electrons

$f(1270)$ meson resonance

Iron nucleua 


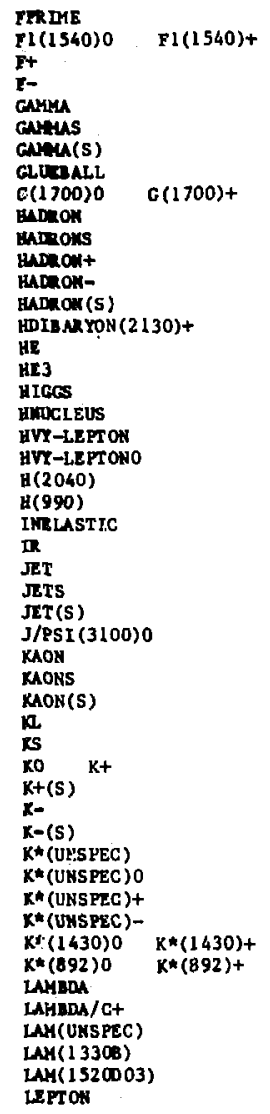

\section{F1(1540)-}

armed atrange meson

two or more gamas

one or pore gamas

\section{G(1720)-}

single hadron, any charge or mass

two or more hadrons

positive hadron

negative hadron

ore or more hadrons

$S=-1$ dibaryon $r$ :onance

hellum-4 nucleus

hellum 3

1883 boson

ypernuc leus

genural heavy leptun

heavy lepton

$\mathrm{I}=0, \mathrm{JP}=4+$ meson resonance

same 25 ANrThING, except elastic excluded Iridium nucleus

jet detected as a whule

two or more jets, each detected as a whole

onc or more jets, each detected as a whule

one kaon or antlkaon of unsjectfled charge

two or more unspecifled kaon.

or more unspectfied kaon

$K$ long

one or more $\mathrm{K}+$

one or more $\mathrm{K}$ -

unspectifled $\mathrm{K}$

unspecified $K^{*}$

unspectfled $K^{\star}$

unspectfied $K^{*}$

$K *(14.30)-$

$K \star(892)-$

charmed baryon

$=0$, $S=1$ baryon resonance

bump at $1330 \mathrm{MeV}$

unspecifled lepton
LI6 LI7

3.NGLI VED

MESON

MESONS

MESON (S)

MESON (UNSPEC)+

MESON (UNSPEC) -

MESON (2950)

MM. GE. 2

MONOFJ

MUON

MUONS

MUON(S)

NE

NEUTRAL.

NEUTRALS

NEUTRA:(s)

NEUTRONS

NEUTRON(S)

NIT 12

NU

NIGLEON

NUCLEONS

NUCLEON (S)

NUCLEUS

NUE

NUMU

NUCAU

$\mathrm{N} * 5 / 2$ (UNS PEC)

$N \star$ (UNSPEC)

$N \star(U N S P E C) 0$

$N^{*}\{$ UNSPEC $\}+$

N(PRMPio)

N(SPECT)

N(UNSPEC)O

N(INS PEC) +

$N(1470 B) 0$

$\mathrm{N}(1470 \mathrm{P} 11) \mathrm{O}$

$\mathrm{N}(1520 \mathrm{~B}) 0$

$N(1520013) 0$

$N(1670015) 0$

$N(1700 B) 0$

o lithium nutel

stable under strong or electromagnetic decay; mass and other quantum nuabers not specified single metan of unepecifled typo

two or more mestons

one or more tresons

neutral meson of unapecified mass

cl, argetl meson of unspectified taes

churge-1 meson of unspectfied mass

buap seen in $p$ pbar p1-

magnealum nucleus

two or more undetected neutral particles

magnetic monopole

any mut or mu-

two or more muons

one or more moons

neutron

geon nucleus

siagle neutral particle

two or more neutral particles

one or more neutral particles

two or more neutrons

one or more neutrons

two or more unspectfied nucleons

one or more unspecteled nucleons

general nucleus

general nucleus

muon neut:r1i10

neutrino asooctared vith tau-

$I=5 / 2, Y=1$ baryon of unapectfied mass and charge

$S=0$ baryon of unspecified mass and 1sospin

$5=0$ baryon of unspecified mass and 1sospin

S= 0 baryon of unspecified mass and 1soopts

a collection of reactions with different numbers of prongs, e.g. 0(prong), 2(prong), 4(prong), etc.

spectator neutron (not number of spectators)

$I=1 / 2, Y=1$ baryon of unspec1fied mass

$N(1470 \mathrm{~B})+$

$I=1 / 2, Y=1$ baryon of unspectified mase

$\mathrm{N}(152 \mathrm{OB})+$

$\mathrm{N}(1520013)+$

$\mathrm{N}(1670 \mathrm{D} 15)+$

$(1700 B)$

oxygen rucluus 
PARTICLE VOCABULARY (CONT'D)

OYEGA

GEGA* (UNSPEC)

OrEF* (UNS PEC)-

or

PH

PHI

PION

PIONS

PION(S)

PIO

PIO(S)

PIt

PItS

PIt

PI-

PI-

$\mathrm{PI}-$ (S)

PRONGS

PRONG (S)

PROTONS

Proton(S)

PSI (UNSPEC)

PSI (3685)

PSI ( 3770 )

PSI (4415)

PT

PT

QUARE.

QUARK(1/3)

QUARR (1/3)

Q(1240-1 400)

Q(1240-1 400)0
RHOPR IME $(1250) 0$

RHOPR IME $(1250) 0$
RHOPR IME $(1550) 0$

RHOPR IHE $(1600) 0$

RHOO RHO+ RHO

SIGuo SIGAAt SIGAA/C(2430)H SIG (UNSPEC) 0 SIG (UNS PEC) SIG(UNSPEC)SIC (I 385P 13)0 $\operatorname{SIG}(16708) 0$ $\operatorname{SIG}(16708)+$

$\operatorname{SIG}(1670 B)-$

\section{meson resonance}

S=-3 baryon

5-3 baryon resonance of unspectf. $d$ isospl. and mass

5m-3 baryon resonance of unspec 1 ed masa

lead nucleus

unspectfied recurrence of the phi

one pion of unspectfled charge

two or more plons

one or more pions

two or mare $10^{\prime} s$

two or more $\mathrm{p}^{1+t^{\prime} \mathrm{s}}$

one charged pion

one ot more $\mathrm{p}^{1+\mathrm{s}}$

two or more pi-'s

one or more $\mathrm{pl}^{-}$'s

two or more prongs

ane or more prongs

one or more protons

unspecified JP=1- charmonium state

platinum nucleus

opectator proton

quzrk of unspecified charge

4uark uf charge $1 / 3$

$2 / 3$

Q(1240-1400)+ Q(1240-1400)-

RHOPR IME (1250)+ RHOPR MIE (1250)-

RHOR ME (15SO)+ RHORE(1550) -

RHO-

SICAA-

11 icnn nucleus

charmed baryon

I-1, Yw0 particle o $E$ unspecified mass

In, $Y=0$ particle of unsperified mas

$I=1, Y=0$ particle of unspecified mass

SIG(1 385P 13)+ SIG(1385P 13)-

$I=1, Y=0$ bump

I $1, Y=0$ bump
SN

STRANGE

STRANGEONIUM

STPANGE (S)

S*(380)

S(1935)0

TA

AU

TAU
TAU+
TAU-

TORONIUM

TRLTH

U

UNSPEC

UPS I (UNSPEC)

UPSI $(10020)$

UES I ( 9460$)$

WESON

MESONO

WT

wO

wo

Wi-

XI 0 XI-

$X I *$ (JNSPEC)

$\mathrm{XI}$ * (UNSPEC) 0

XI (UNSPEC)

$X I$ (UNS PEC) 0

XI (UNSPEC)-

$X I(1530 P 13)$

$\mathrm{XI}(1620) 0$

$\mathrm{XI}(1940) 0$

$\mathrm{X}(2830)$

$Y \star($ UNSPEC)

$Y \star(U N S P E C)+$

$Y *(U N S P E C)-$

20

Z* (UNSPEC) 0

\section{PARTICLE VOCABULARY (CONTD)}

tIn nucleus

unspecteted strange particle

me. on those quark content is doninantly s-sbar, such as the phi

one or more unspecifed strange particles

Intermediate scalar boson

intermediate scalar boson

p1-pI or K-Kbar S-wave

$s(1935)+\quad S(1935)-$

tritium nucleus

tantalum nurleus

heavy lepton

positive heavy lepton

negative heavy leptor

tItanium nucleus

rop-antitop state

generic name for any particle with naked truth uranturn nucleus

particle of unspecified type

unspecified upsilon particle

one or more unspecified neutral strange particle

decays

vector meson of unspectfled mass and charge

vector meson of unspecified mars

Intermediate vector boson

tungsten nucleus -- note nawe is not same as

chemical symbol

intermediate vector boson

intermediate vector boson

intermediate vector boson

S=-2 baryon of unspecified mass

$I=1 / 2, S=-2$ baryon of unspecified mass $X I(1530)$ $X I(1820)-$ XI (1940)-

JPm0 - sharmonium state

$S=-1$ barvon of unspectfied 1sospin and mass $S=-1$ baryon of unspecified 1 sospin. and mass $S=-1$ baryon of unspecified 1 aospin and mass $S=-1$ baryon of unspecified isospin and rass neutral weak gauge boson

exotic $x=2$ baryon of unspecified mass 


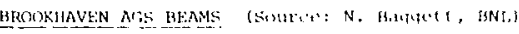

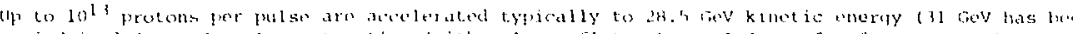

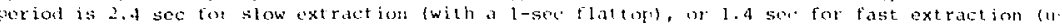

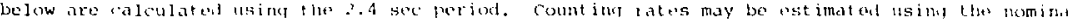

sbtaineds. At $2 H .5$ (icV, the for neittrino beams). Fluxes

\begin{tabular}{|c|c|c|c|c|c|c|c|}
\hline & $\begin{array}{l}\text { Pomcutusu } \\
\text { rumic }\end{array}$ & "州么 & Frominct iom & 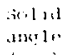 & $\begin{array}{l}\text { Hesan } \\
\text { lementh }\end{array}$ & & $\begin{array}{l}\text { Flux in lomsants } \\
\text { per sec por lold }\end{array}$ \\
\hline $\mathrm{am}$ & $(1, \cdots \times<)$ & {$[\because]$} & (amllt: $\left.r^{n}\right)$ & (msti) & $(\mathrm{m})$ & lartioles & protants on target \\
\hline
\end{tabular}

\section{$\therefore$ inv/c) commont \\ $4 \quad$ Isually $2 \times 1012$ H"lp on target: $\pi / \mathrm{K}-$ \\ in $\mathrm{k}$ beam}

Th maltiparticle spect rometer

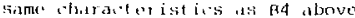

跑

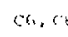

15 $+$

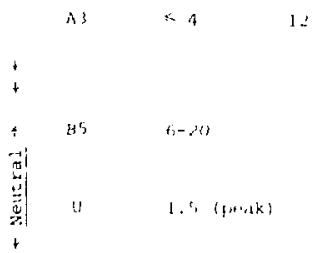
$n^{+}=\pi^{-}$

$\begin{array}{cc}\mathrm{k}^{+} / \mathrm{K}^{-} & \cdots 121 \% \\ \overline{5} & 3 \times 10^{4} / 3 \times 20^{-1}\end{array}$

$3 \times 10^{4}$

$1000 / 5600$

14

$6 \times 13^{5}$

(0) 117

1.5

$10^{4} / 30010$

$2500 / 700$

$1.5 \times 10^{5} / 200$

$1 \times 11^{4}$

$1000 / 4100$

$1 \times 10^{6} / 30$

$10^{5} ; 3 \times 10^{17}$

$4 \times 10^{4} / 4 \times 10^{4}$

$\times 1 n^{4} / 4 \times 10^{3}$

$10^{6} / 2 \times 10^{5}$

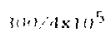

$\therefore 2 \times 10^{4}$

$11)^{3}<1 \times 110^{6}$

prer ...."
To nuiliparticle spectroncter:

$10^{12} \mathrm{pPF}$

.0

Usually $2 \times 10^{12} \mathrm{ppp}$

16

Usualty $2 \times 10^{12} \mathrm{ppp}$ $\mu / \pi$ - 3q it $\pi$ beam

TYically $10^{11}$ ppp; al ternates with Al

Typicaly $10^{10}$ PPP

Typically $9 \times 10^{12} \mathrm{FPp}$. flux averayed over $0.7 \mathrm{~m}$ radius 


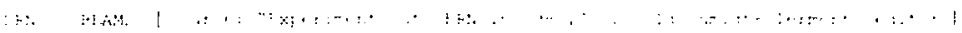

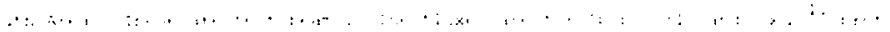

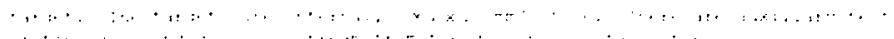

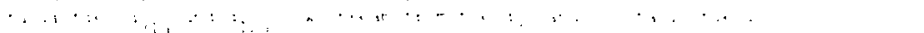
$\therefore, \quad \because, \ldots, \ldots$

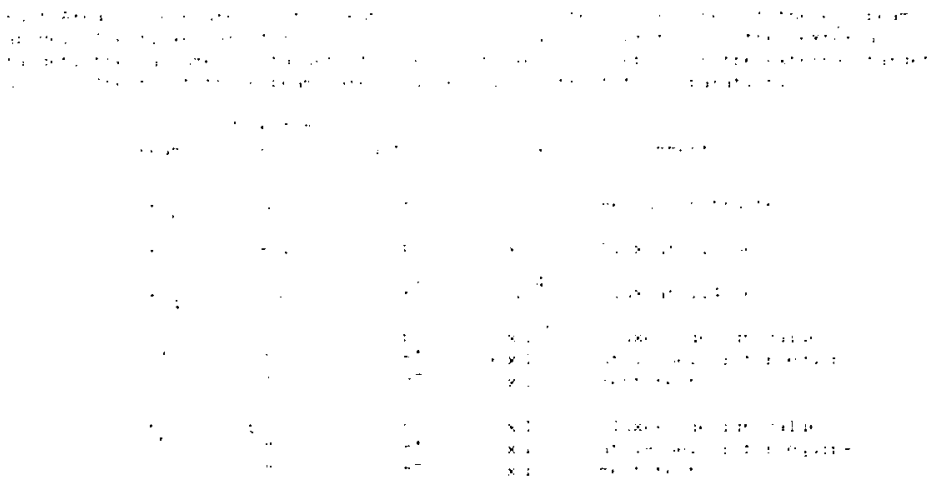


25

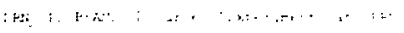

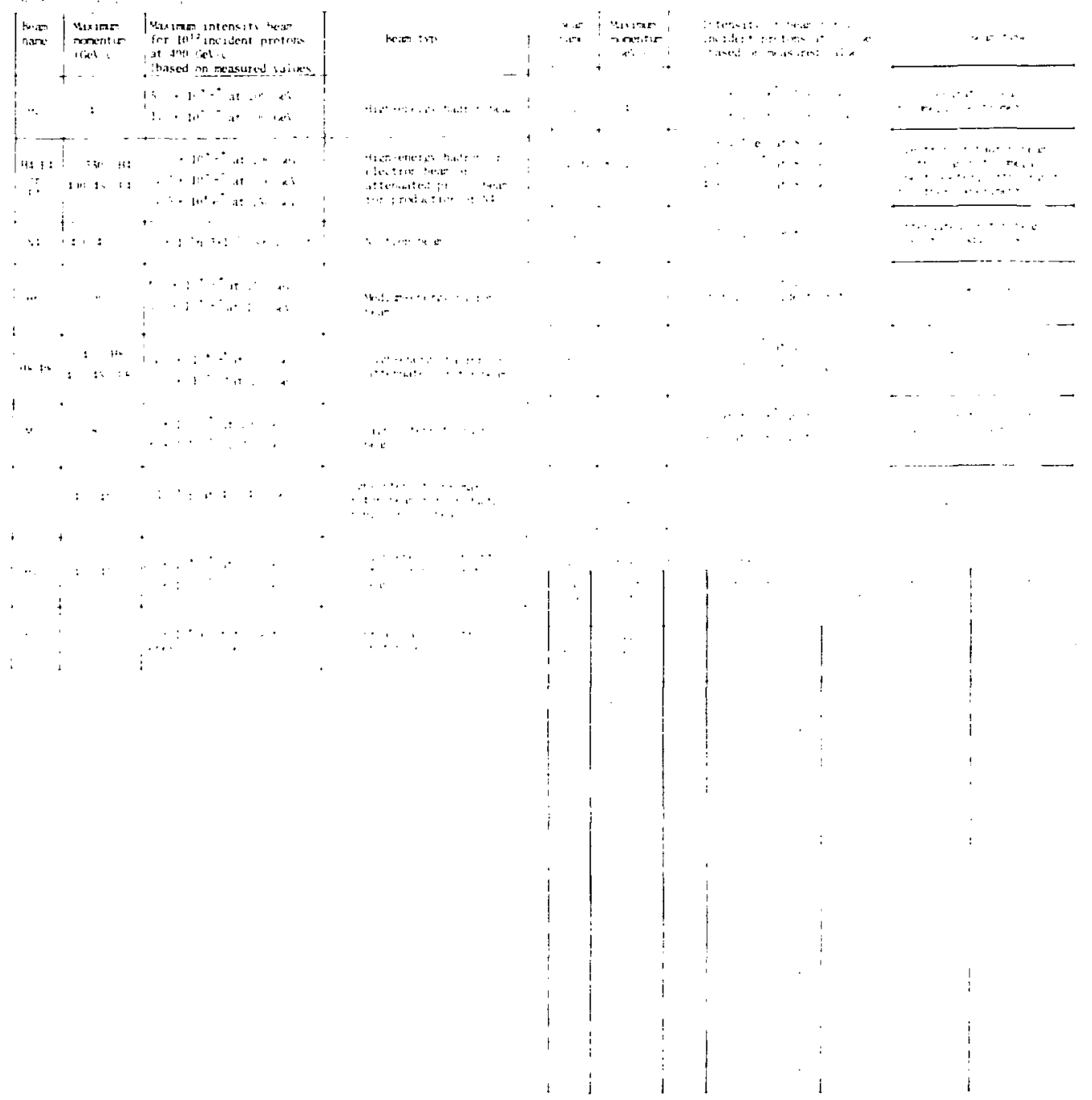




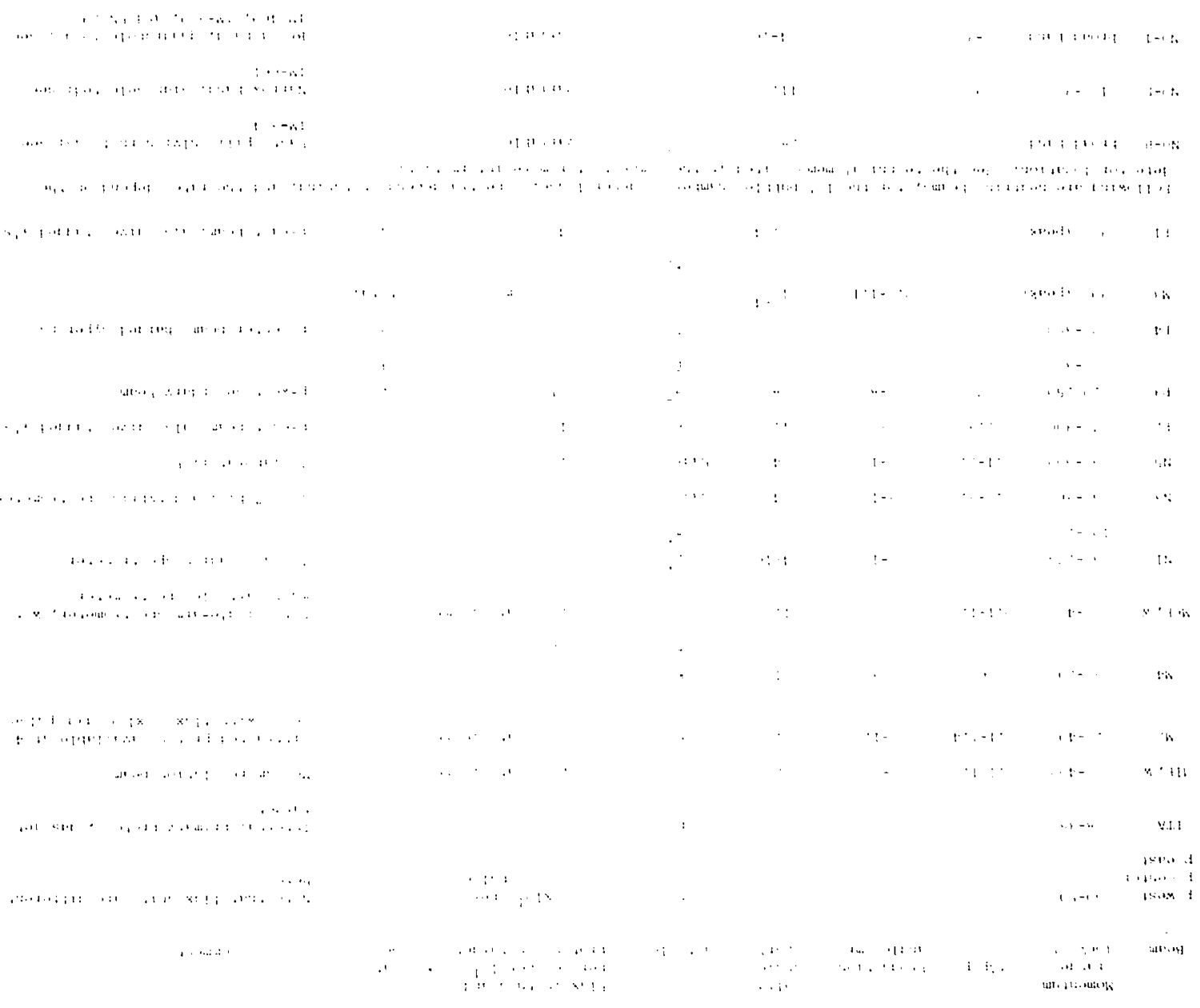


27

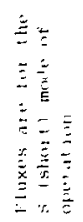

$$
\begin{gathered}
0 \\
\vdots \\
\vdots \\
\vdots
\end{gathered}
$$

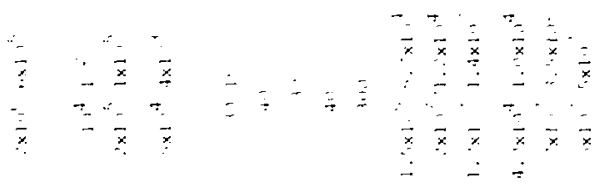

$\overline{\bar{x}} \stackrel{\bar{x}}{\bar{x}}$

$-\quad \cdot$

$$
\therefore=
$$

$\bar{z}$

$\vdots \quad \vdots \quad \div \quad$

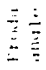

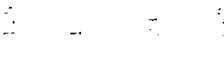

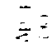

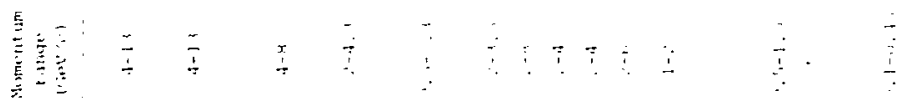

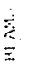

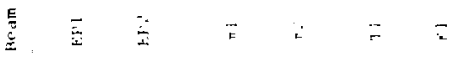


28
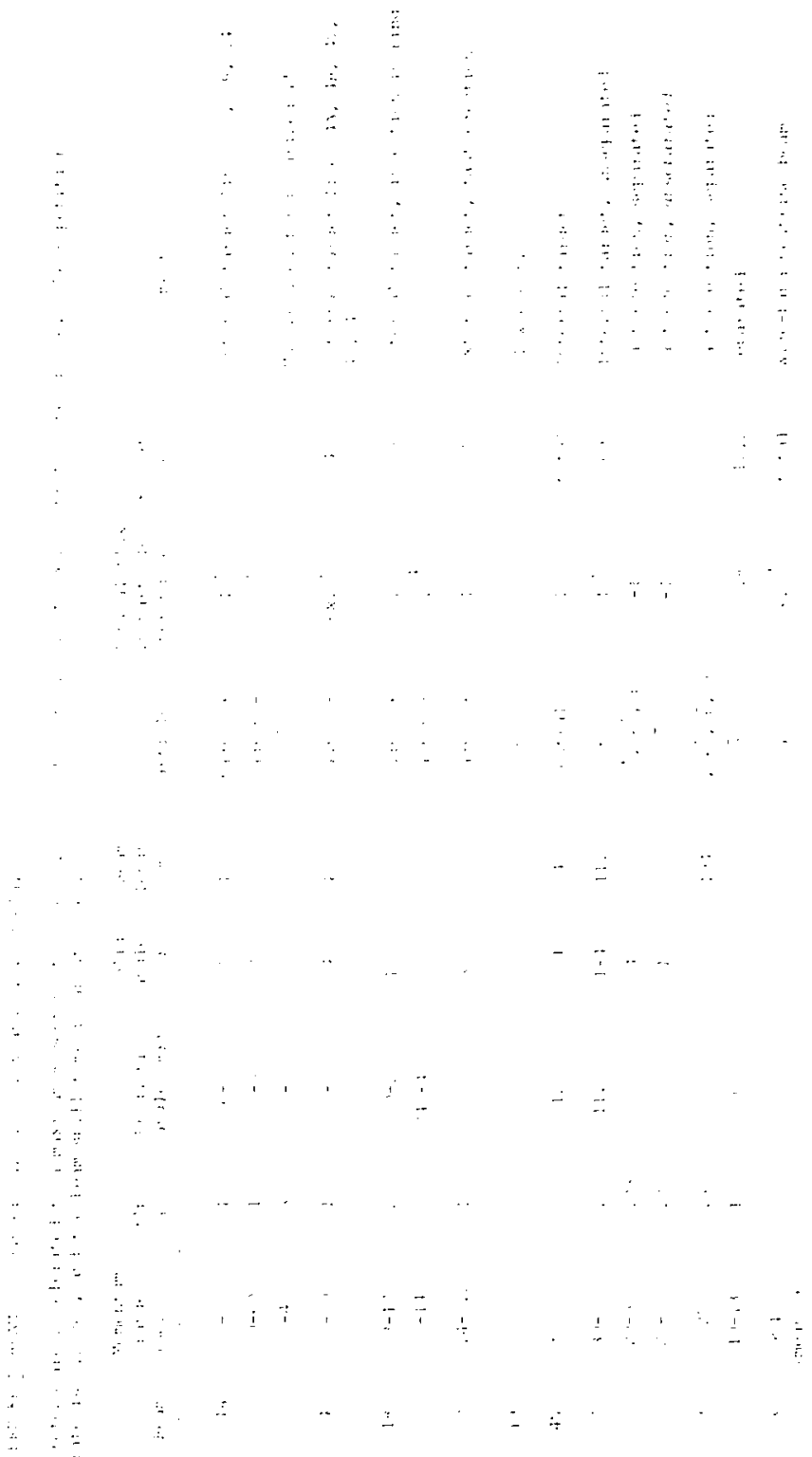
SLAC REMMS (Source: T. Fiecguth, SIN")

\begin{tabular}{|c|c|c|c|c|c|c|}
\hline $\begin{array}{l}\text { Accelerutor } \\
\text { moide }\end{array}$ & Particles & $\begin{array}{l}\text { Moment a } \\
(\text { Gev/e) }\end{array}$ & $\begin{array}{l}\text { lartioles } \\
\text { per pulse }\end{array}$ & 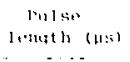 & $\begin{array}{l}\text { Kelped it icm } \\
\text { rate (Hz) }\end{array}$ & 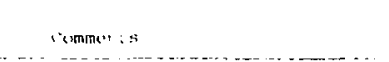 \\
\hline Normal & $\mathrm{e}^{t}$ & $\begin{array}{l}\leqslant 3.3 .5 \\
\leqslant 15.0\end{array}$ & $\begin{array}{l}\$ 5 \times 10^{11} \\
\leqslant 2 \times 10^{10}\end{array}$ & $\begin{array}{l}1.11 \\
1.16\end{array}$ & $\begin{array}{l}=8,00 \\
=(x)\end{array}$ & \multirow{2}{*}{ 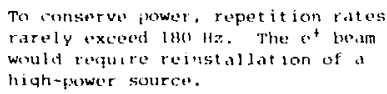 } \\
\hline SLFD & $e^{-}$ & $\leqslant 33.5$ & 1011 & $0 .$. & $=360$ & \\
\hline
\end{tabular}

\begin{tabular}{|c|c|c|c|c|c|}
\hline $\begin{array}{l}\text { Colliding } \\
\text { beams }\end{array}$ & Particles & $\begin{array}{c}\text { C.m. energy } \\
\text { (GeV) }\end{array}$ & $\begin{array}{l}\text { Peak luminosity } \\
\left(\mathrm{cm}^{-} \sec ^{-1}\right)\end{array}$ & $\begin{array}{l}\text { Average } 1 \text { umanosity } \\
\left(\mathrm{cm}^{-2} \mathrm{sec}^{-1}\right)\end{array}$ & cumments \\
\hline SPEAR & $e^{+} e^{-}$ & $2 \cdot 1.4$ & $2 \times 10^{31}$ at $3.2 \times 3 \times 4$ & $13 \times 10^{30}$ & SPEAR has 2 interaction regions, PF, 6 . \\
\hline PF:P & $e^{\prime} e^{-}$ & $H-36$ & $7 \times 10^{30}$ it $24 \mathrm{GeV}$ & $3 \times 10^{30}$ & 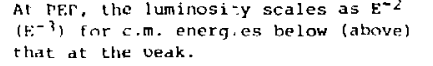 \\
\hline
\end{tabular}

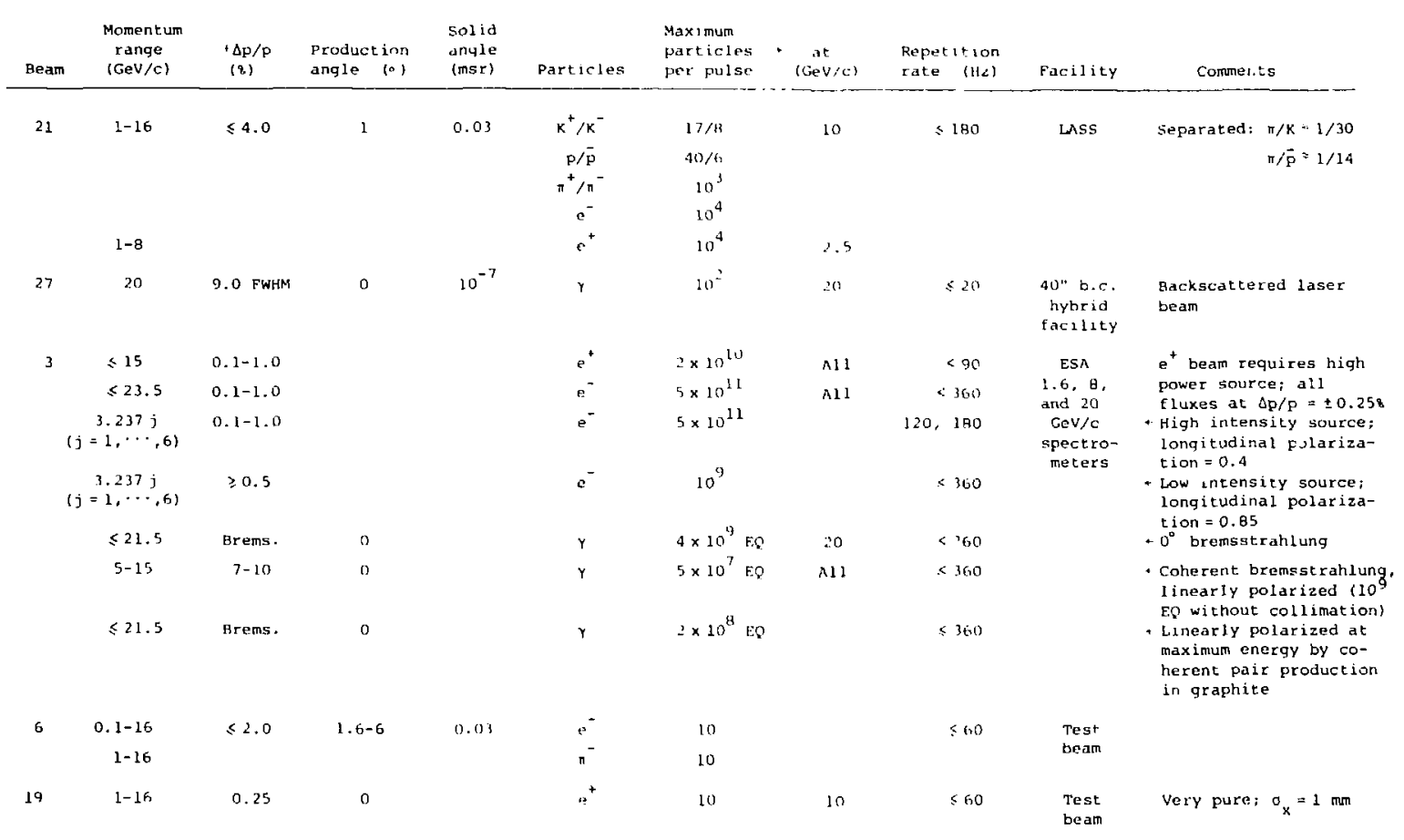

